\title{
The Nuclear Collective Motion
}

\section{Witold Nazarewicz}

Department of Physics, University of Tennessee, Knoxville, Tennessee 37996

Physics Division, Oak Ridge National Laboratory, Oak Ridge, Tennessee 37831 Institute of Theoretical Physics, University of Warsaw, ul. Hoża 69, PL-00-681

Warsaw, Poland

\begin{abstract}
Current developments in nuclear structure are discussed from a theoretical perspective. First, the progress in theoretical modeling of nuclei is reviewed. This is followed by the discussion of nuclear time scales, nuclear collective modes, and nuclear deformations. Some perspectives on nuclear structure research far from stability are given. Finally, interdisciplinary aspects of the nuclear many-body problem are outlined.
\end{abstract}

\section{Introduction}

The atomic nucleus is a fascinating many-body system bound by strong interaction. The building blocks of a nucleus - protons and neutrons - are themselves composite aggregations of quarks and gluons governed by quantum chromodynamics (QCD) - the fundamental theory of strong interaction. Nuclei are exceedingly difficult to describe; they contain too many nucleons to allow for an exact treatment and far too few to disregard finite-size effects. Figure 1 shows

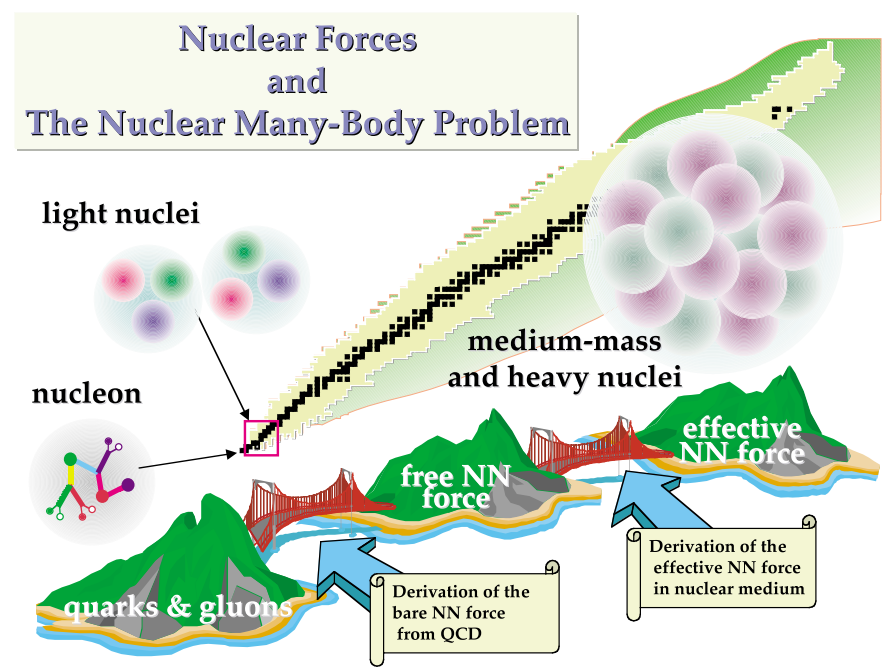

Fig. 1. From the QCD vacuum to heavy nuclei: the intellectual connection between the hadronic many-body problem (quark-gluon description of a nucleon) and the nucleonic many-body problem (nucleus as a system of $Z$ protons and $N$ neutrons). From Ref. [1]. 
the main challenges in our quest for understanding the nucleus. Studies at relativistic energies probe the domain of QCD; they reveal the nature of quark and gluon dynamics. Studies at lower energies probe the structure and dynamics of nuclei. The bridges illustrate major physics questions pertaining to the nucleus: the nature of hadrons, the understanding of the bare nucleon-nucleon interaction in terms of the quark-gluon dynamics, and the understanding of the effective interactions in heavy nuclei in terms of the bare force.

In this talk, I intend to review - rather briefly - a number of issues relevant to the nuclear many-body problem. Firstly, Sec. 3 covers the enormous progress that has happened in theoretical nuclear structure during recent years. (For a general overview of nuclear science, the reader is encouraged to study the recent report [2].) Some spectacular examples of the nuclear collective motion are presented in Sec. 4. The concept of nuclear deformation, i.e., anisotropic nuclear mean field, is one of the most useful and important building blocks of the unified model. In Sec. 5, several different views on the microscopic origin of nuclear deformation are presented. The common denominator is the interplay between the symmetrybreaking particle-vibration interaction and the symmetry-restoring pairing force.

One of the frontiers of today's nuclear science is the "journey to the limits" of atomic charge and nuclear mass, of neutron-to-proton ratio (see Sec. 7). The tour to the limits is not only a quest for new, exciting phenomena, but the new data are expected, as well, to bring qualitatively new information about the fundamental properties of the nucleonic many-body system, the nature of the nuclear interaction, and nucleonic correlations at various energy-distance scales.

Section 8 discusses interdisciplinary aspects of the nuclear many-body problem, and Sec. 9 contains conclusions of these lectures.

\section{The Territory}

The nuclear landscape, the territory of nuclear structure, is shown in the top portion of Fig. 2. Moving away from the valley stable nuclei by adding either protons or neutrons, one enters nuclear "terra incognita" which is bordered by the particle drip lines where the nuclear binding ends. The nuclei beyond the drip lines are unbound to nucleon emission. Examples of such systems are proton emitters - narrow resonances beyond the proton drip line which exist due to the confining effect of the Coulomb barrier [3]. An exciting question is whether there can possibly exist islands of stability beyond the neutron drip line. One such island is, of course, a neutron star which exists due to gravitation. So far, calculations for light neutron drops have not produced permanent binding $[4,5]$. However, it has been suggested recently [6] that areas of stability can appear in heavier nuclei as a result of shape coexistence/isomerism.

The vast territory of nucleonic matter is shown in Figure 3 which illustrates various domains of nuclear matter. The range of neutron excess, $(N-Z) / A$, in finite nuclei is from about -0.2 (proton drip line) to 0.5 (neutron drip line). The new-generation radioactive beam facilities will provide a unique capability for accessing the very asymmetric nuclear matter and for compressing neutron-rich 


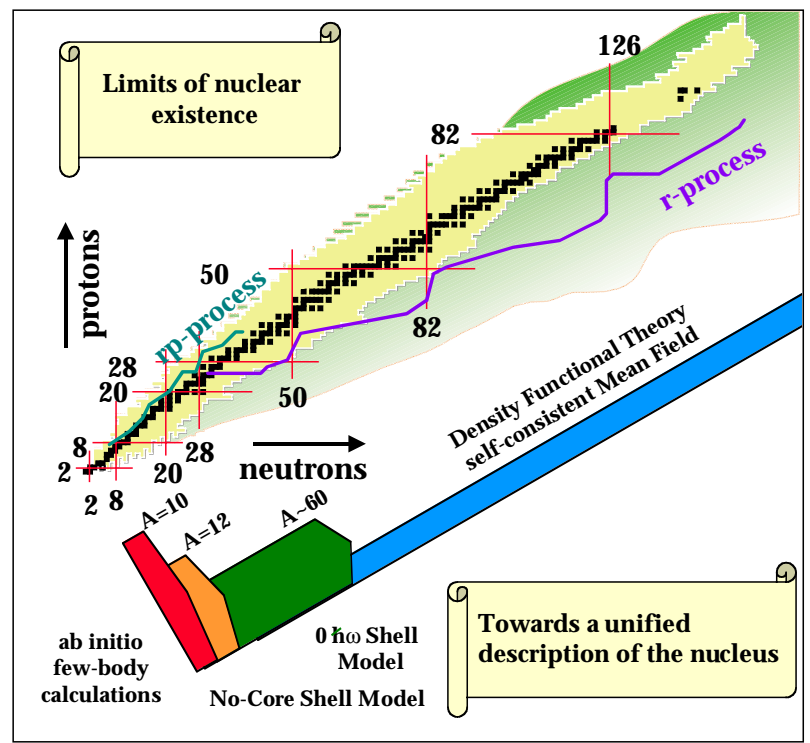

Fig. 2. Top: Nuclear landscape. Bottom: Various theoretical approaches to the nuclear many-body problem.

matter approaching density regimes important for supernova and neutron star physics.

\section{The Nuclear Many-Body Problem: Towards the Unified Description of the Nucleus}

The common theme for the field of nuclear structure is that of the nucleonnucleon $(N N)$ interaction which clusters nucleons together into one composite system. Figures 1 and 2 illustrate, schematically, our main strategy in the quest for understanding the nucleus in the context of the hadronic and nucleonic manybody problem.

The free $N N$ force can be viewed as a residual interaction of the underlying quark-gluon dynamics of QCD, similar to the intermolecular forces that stem from QED. The low-energy interaction between nucleons has a complicated spin-isospin dependence dictated by the hadron's substructure. One of the main challenges of nuclear science, indicated by the first bridge in Fig. 1, is the derivation of a nucleon-nucleon $(N N)$ interaction from the underlying quark-gluon dynamics of QCD. Experimentally, the $N N$ force can be studied by means of $N N$ scattering experiments. Examples of phenomenological parameterizations based on the $N N$ scattering data are the Bonn [8,9], Nijmegen, Reid 


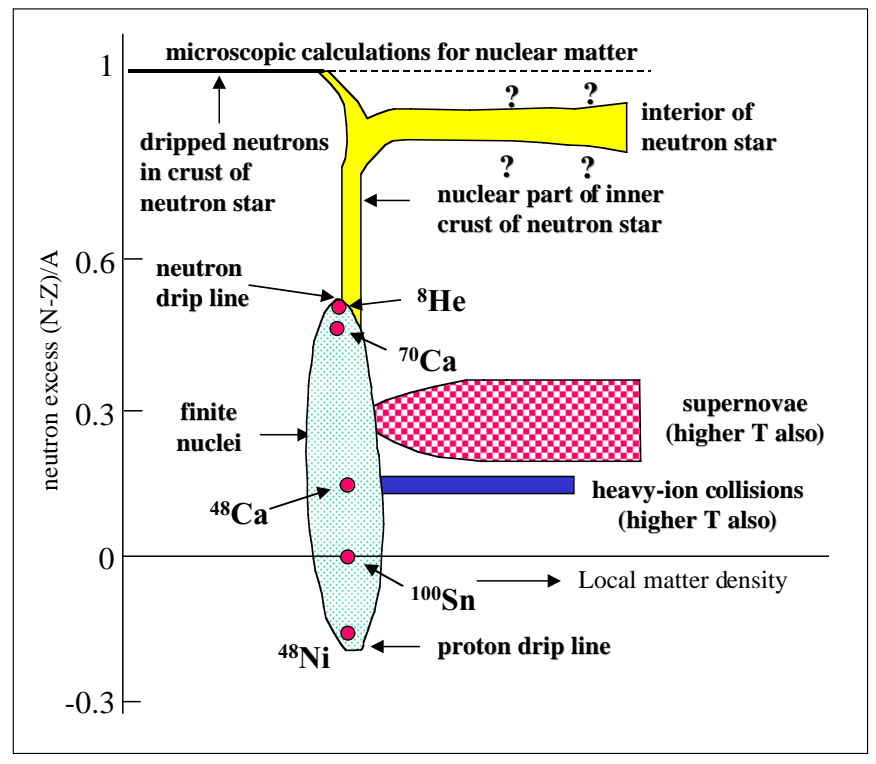

Fig. 3. Diagram illustrating the range of densities and neutron excess of importance in various contexts. (Based on Ref. [7].)

[10], Paris [11], and Argonne [12] potentials. While the long-range part of these free (but still effective!) $N N$ forces is well described by one-pion exchange, their short-range behavior is purely phenomenological. Here the quark-gluon degrees of freedom must be considered explicitly. (It is believed that the short-range part can be well accounted for by a simple one-gluon exchange potential and the Pauli principle $[13,14]$.)

How to tackle the problem of $A$ strongly interacting nucleons? The general theoretical strategy is illustrated in Fig. 4. The starting point is, of course, the exact solution of the $A$-body Schrödinger equation (or relativistic field equations) with the bare $N N$ force. Today, such ab initio calculations can be performed for very light nuclei, but still it is a very difficult task. Firstly, the corresponding dimensions are huge. Secondly, the bare $N N$ force is very complicated (e.g., it contains tensor terms and, often, non-local terms) and the form of higher-order interactions, such as a three-body force $(N N N)$, is not well known.

The state of the art is marked by the ab initio Green's Function Monte Carlo (GFMC) calculations [15] which have recently reached $A=10$. Figure 5 shows the results of GFMC calculations by the Argonne-Los Alamos-Urbana collaboration of the excitation spectrum of $A=4-9$ nuclei using the GFMC method. The variational Monte Carlo calculations with a free $N N$ force have been carried out for relatively heavy systems such as ${ }^{16} \mathrm{O}[17]$. Another ab initio method, which is 


\section{The Nuclear Many-Body Problem}

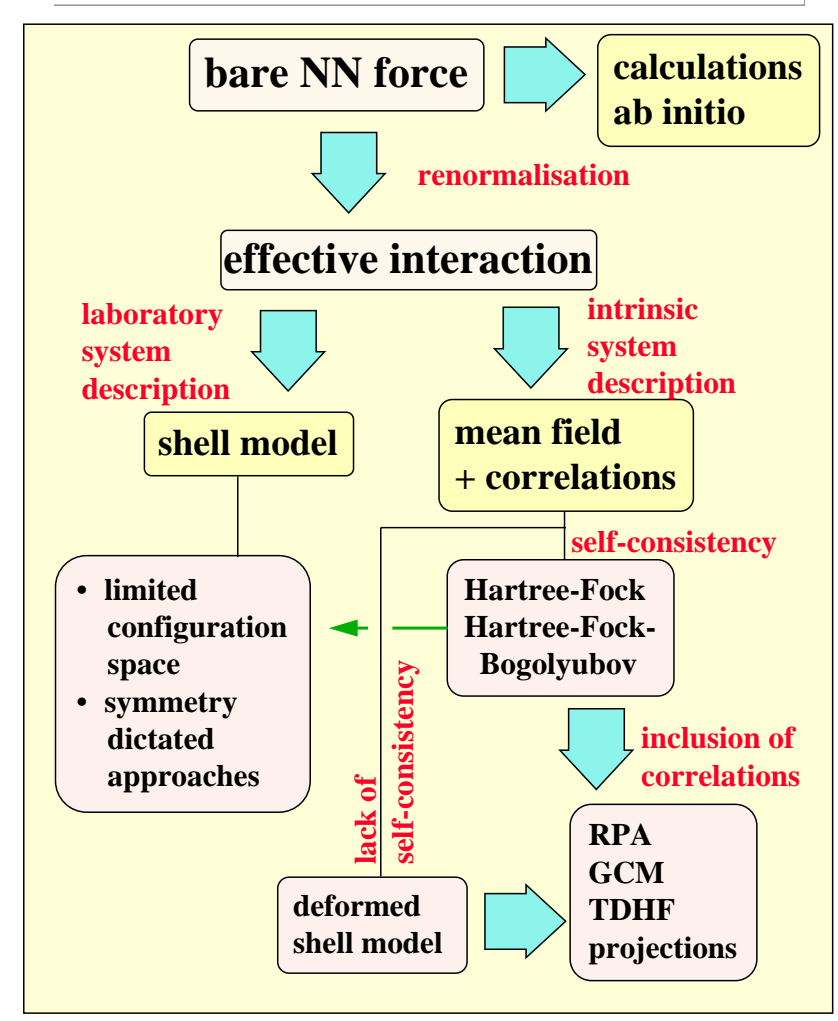

Fig. 4. The nuclear many body problem - various theoretical strategies.

currently undergoing a renaissance is the coupled-cluster [or "exp(S)"] method [18-20] (see Refs. [21-23] for recent applications). In the parallel development $[24,25]$, ab initio calculations describing the scattering of few-body systems have brought new insights into the nature of the three-nucleon force.

While the very light nuclei can nowadays be described as $A$-body systems bound by a free $N N$ force augmented by a $N N N$ force, the conceptual framework of larger nuclei is still that of the independent particle model. Here, the basic assumption is that the nucleons are moving almost independently in a mean potential obtained by averaging out the interactions between a single nucleon and all remaining $A-1$ protons and neutrons. This picture is only a first approximation; it is modified by the presence of the residual interaction between the nucleons. The "effective" $N N$ interaction in the heavy nucleus, used to determine the mean potential, differs considerably from the free $N N$ force. In principle, it should be obtained by means of the complicated Brückner renormalization procedure which corrects the free $N N$ interaction for the effects due to the nuclear 


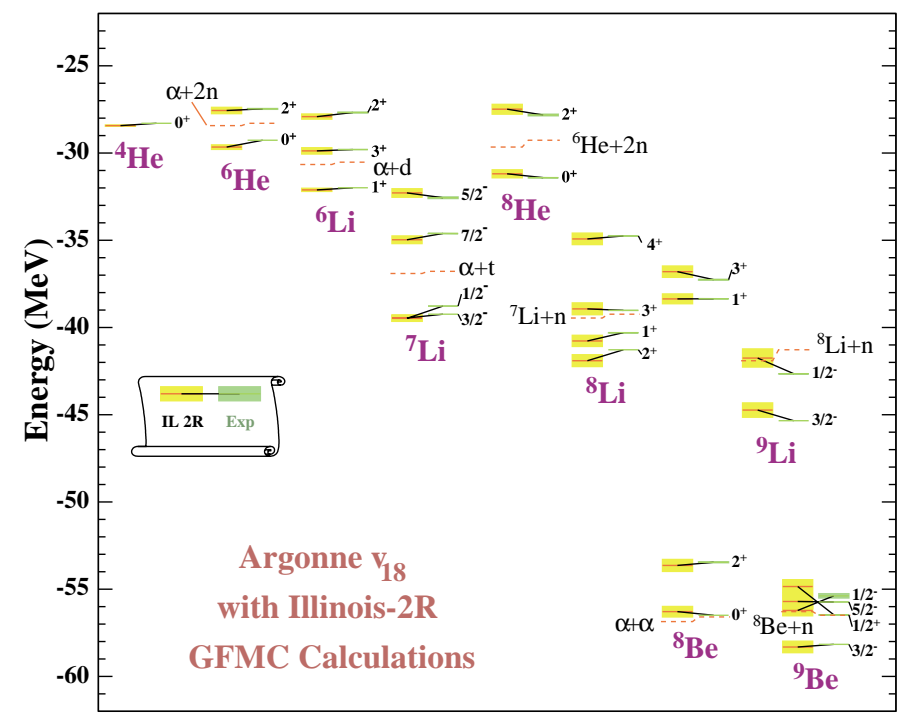

Fig. 5. GFMC calculations of excitation spectra of $A=4-9$ nuclei using Argonne $v_{18}$ $N N$ force and Illinois-2R $N N N$ force. (From Ref. [16].)

medium [26-28]. (In practice, however, effective forces are often determined by a fit to experimental data, or by extracting interaction matrix elements from the data.) This challenging task is represented by the second bridge in Fig. 1. Many features of the effective interaction such as short range; strong dependence on spins, isospins, and relative momenta of interacting nucleons; and the reduction of mass in the nuclear interior have been extracted from experimental data. Having determined the effective force, the two most commonly used strategies, represented by a forking in Fig. 4, are (i) that of the nuclear shell model and (ii) the mean-field strategy.

In the nuclear shell model, the effective two-body Hamiltonian is diagonalized in the limited configuration space. Here, the practical limitation is the size of the Hilbert space considered. Often, the realistic shell-model Hamiltonian can be further approximated by replacing it with a Hamiltonian which can be diagonalized exactly using group theoretical techniques [29-33]. The wave function of the nuclear shell model is an eigenstate of fundamental symmetry operators; it has good parity, angular momentum, and isospin. From this point of view, it is a laboratory system wave function.

The recently developed no-core shell model, employing the effective interaction calculated (in the large configuration space) from the $N N$ force, has recently reached ${ }^{12} \mathrm{C}$. Figure 6 shows the result of the no-core shell-model calculations [34] for positive and negative-parity states of ${ }^{12} \mathrm{C}$. In a parallel development, Bloch-Horowitz equations have been solved for very light systems [35], and an effort has been under way [36] to marry the numerical methods of the shell model 


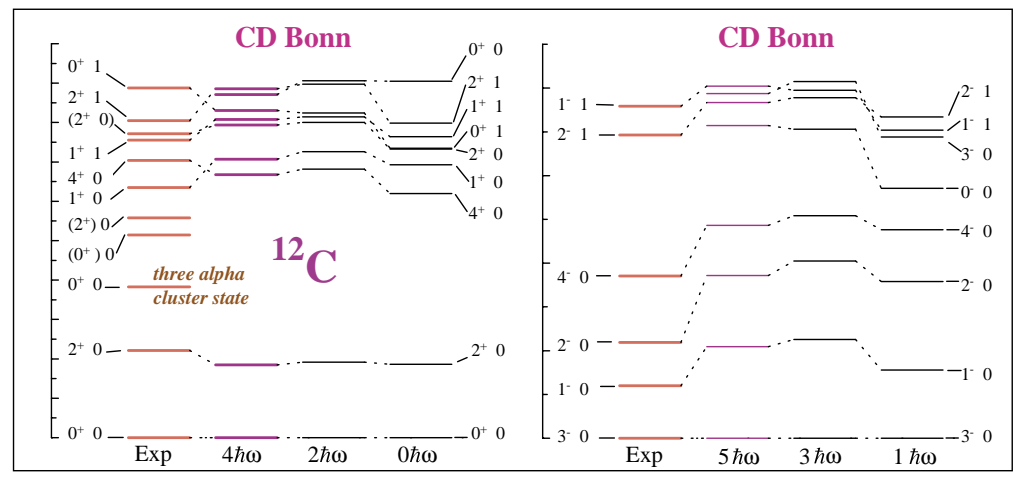

Fig. 6. No-core shell-model calculations of the excitation spectrum of ${ }^{12} \mathrm{C}$ using the CD Bonn $N N$ interaction. (From Ref. [34].)

with the tools of effective theory [37] to generate effective interactions and effective operators. (In this approach the hard-core contribution is summed to all orders analytically.)

There has been substantial progress in the area of the traditional shell model [38]. In 1971, Whitehead and Watt [39] succeeded in performing shell-model calculations for ${ }^{24} \mathrm{Mg}$ in the full $s d$ shell (with the dimension of the model space approaching 30,000 ). Today, this can be done in a few seconds on a modern workstation. Traditional shell-model techniques make it possible to approach the collective nuclei from the $p f$ shell; the calculations involve model spaces with dimensions of several millions. However, progress in this area is going to be very slow due to exploding dimensions when increasing the number of valence nucleons [40,41] (see Fig. 7). In spite of this, the conventional shell-model calculations employing realistic $N N$ interactions $[42,43]$ are becoming more and more efficient in handling large configuration spaces. The state-of-the-art shell-model studies of Gamow-Teller distributions of $A=45-65$ nuclei [44], electron capture and beta-decay rates in the $p f$ nuclei [45], spectroscopic studies of $A=50-52$ isobaric chains [46], and of quadrupole and magnetic moments of the Fe isotopes [47] set the new standard in this area, although future progress is strongly limited by present-day computer resources.

One actively pursued alternative is to truncate the configuration space by applying the projected self-consistent quasiparticle basis [48,49]. Another family of novel shell-model techniques is based on the Monte Carlo method [50]. Applications of the Monte Carlo shell model have been remarkably successful in describing many structural properties of medium-mass and heavy nuclei [51$54]$ where the dimensions of the model space reach $10^{20}$. The approach adopted by the Tokyo group [53,55] is a mixture of the projected shell model and the Monte Carlo shell model. It was applied to studies of deformed structures in the doubly-magic nucleus ${ }^{56} \mathrm{Ni}$. 


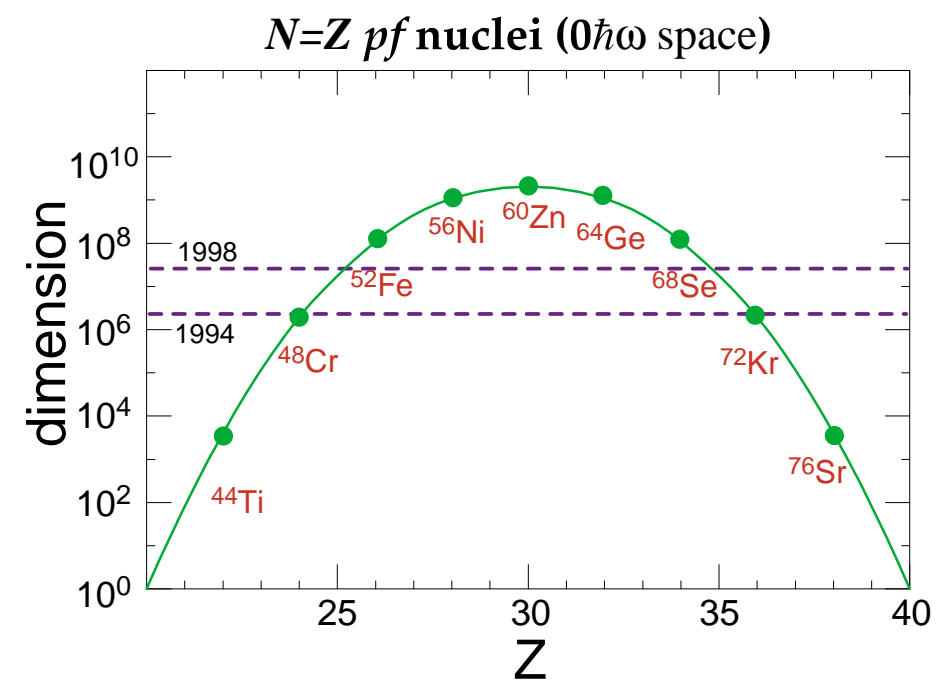

Fig. 7. Dimension of the shell-model configuration space for even-even $N=Z$ $(20 \leq Z \leq 40)$ nuclei. It is assumed that the shell-model calculations are performed in the full $p f$ shell in the $M$ scheme (here $M=0$ ) (courtesy of T. Otsuka).

That the conventional shell model fails at short distances, due to the presence of short-range correlations, has been known for quite some time. Recently, ${ }^{16} \mathrm{O}\left(\mathrm{e}, \mathrm{e}^{\prime} \mathrm{pp}\right){ }^{14} \mathrm{C}$ two-proton knock-out data $[56,57]$ offered an opportunity to study one- and two-body currents and to discriminate between long-range and short-range correlations [58]. Such studies are extremely important for understanding in-medium effects in nuclear matter. Another complementary piece of data, coming from the decay studies, that is relevant to the question of twonucleon correlations, was the recent observation [59] of the simultaneous emission of two protons from a resonance of ${ }^{18} \mathrm{Ne}$. This new mode of nuclear decay was predicted in the $1960 \mathrm{~s}$, but until recently experimental efforts have found sequential emission of single protons though an intermediate state. A key remaining question is whether the two protons, as they leave the nucleus, are closely coupled together to form ${ }^{2} \mathrm{He}$, or are emitted almost independently in a direct three-body breakup ("democratic" decay). Further studies of this phenomenon will shed new light on the nature of nucleonic superconductivity. (See Ref. [60] for recent theoretical developments.)

Despite the exciting progress in shell-model approaches, their applications to very heavy systems are still beyond our reach. Besides, for nuclei with many valence particles, the concept of valence nucleons is less useful, and the valence and inner-shell nucleons have to be treated on an equal footing. To carry out the microscopic, consistent in-medium renormalization for heavy nuclei is a difficult task. Consequently, theories and methods have been developed which use effec- 
tive interactions or effective Lagrangians. Among them are the self-consistent methods based on the density-dependent effective interactions, which by now have achieved a mature state of development, as well as those based on relativistic meson-nucleon Lagrangians which have reached the state where detailed studies of results and readjustment of basic parameters are now possible.

In the strategy of the mean-field theory, the main assumption is that the many-body wave function can be - to zero order - approximated by that of independently moving quasiparticles. Together with the variational principle applied to the effective density-dependent Hamiltonian, this leads to the Hartree-Fock (HF) or Hartree-Fock-Bogolyubov (HFB) equations [28]. In spite of the very simple form, the independent-quasiparticle wave function is a highly correlated state. In the mean-field theory, self-consistency is automatically guaranteed by the equations of motion. That is, the same wave function which generates the mean field is an eigenstate of the mean-field Hamiltonian. Since the mean-field Hamiltonian often breaks symmetries present in the laboratory system, the HFB state is an intrinsic wave function, which - in general - is not an eigenstate of angular momentum, parity, and particle number operators. (The microscopic mechanism responsible for the symmetry-breaking is discussed below in Sec. 5.) By restoring the intrinsically broken symmetries, one takes into account correlations going beyond the mean-field description. This can be done by various theoretical techniques such as the Random Phase Approximation (RPA), the Generator Coordinate Method (GCM), or various projection methods.

The mean-field approaches have achieved a level of sophistication and precision which allows analyses of experimental data for a wide range of properties and for arbitrarily heavy nuclei [61-64]. For instance, a self-consistent mass table has been recently developed [65] based on the Skyrme energy functional. The resulting rms error on binding energies of 1700 nuclei is around $700 \mathrm{keV}$, i.e., is comparable with the agreement obtained in the microscopic-macroscopic approaches. Figure 8 displays two-neutron separation energies for the Sn isotopes calculated in several state-of-the-art self-consistent mean-field models based on different effective interactions and, in the inset, those obtained with phenomenological mass formulae. All these models nicely describe the existing experimental data; some interesting deviations are seen when approaching the proton drip line. This figure nicely illustrates difficulties with making theoretical extrapolations into neutron-rich territory. Clearly, the differences between forces and mass formulae are greater in the region of "terra incognita" than in the region where masses are known. As seen in Fig. 8, the position of the neutron drip line for the Sn isotopes depends on the model used. Therefore, the uncertainty due to the largely unknown isospin dependence of the effective force gives an appreciable theoretical "error bar" for the position of the drip line. Unfortunately, the results presented in Fig. 8 do not tell us much about which of the forces discussed should be preferred since one is dealing with dramatic extrapolations far beyond the region known experimentally. However, a detailed analysis of the force dependence of results may give us valuable information on the relative importance of various force parameters. 


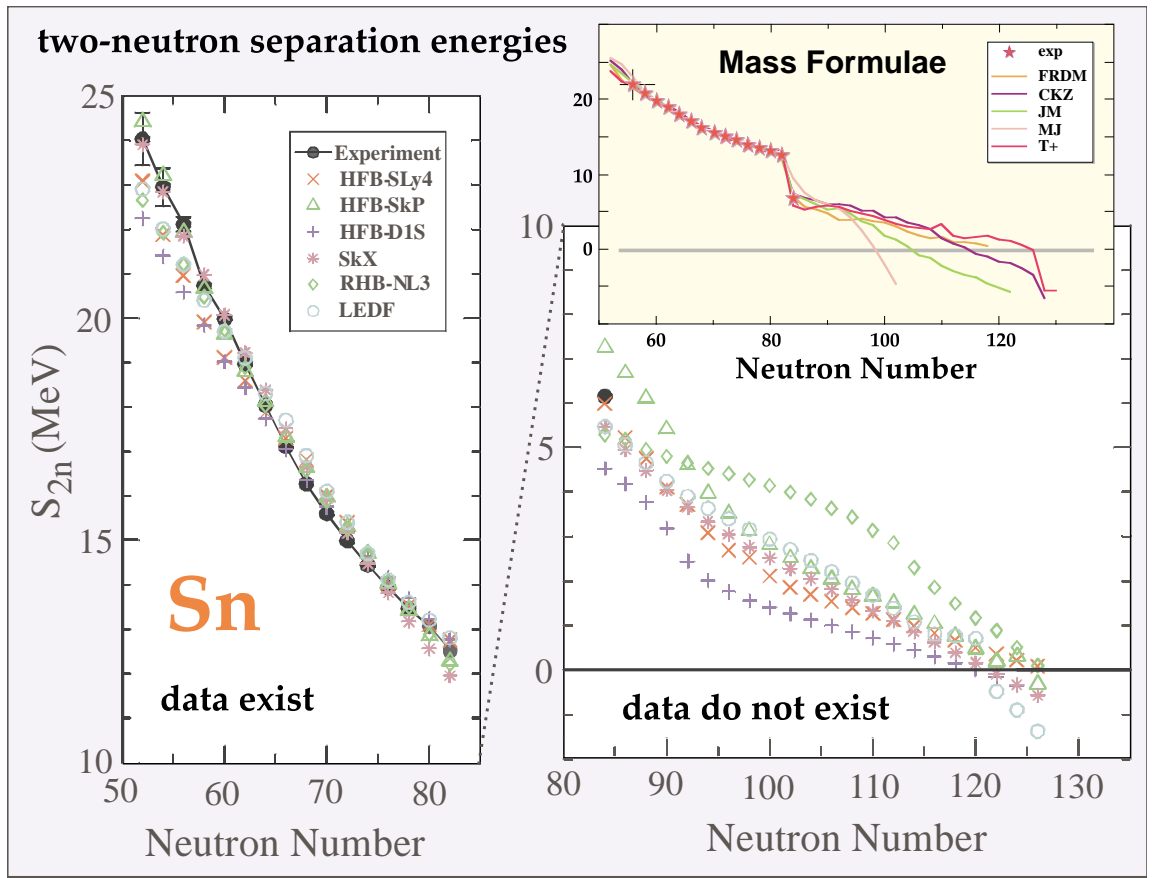

Fig. 8. Predicted two-neutron separation energies for the even-even Sn isotopes using several microscopic models [Hartree-Fock-Bogolyubov (HFB); local energy-density functional model (LEDF); relativistic Hartree-Bogolyubov (RHB)] based on effective nucleon-nucleon interactions and obtained with phenomenological mass formulas (shown in the inset at top right). Taken from Refs. [66,67] and references quoted therein.

Among other recent developments in theoretical nuclear structure, particularly important is the elegant explanation of the pseudo-spin symmetry of the nuclear single-particle spectra proposed thirty years ago $[68,69]$. Surprisingly, as demonstrated by Ginocchio [70], the roots of the pseudo-spin can be traced back to the symmetry of the Dirac equation (see also Refs. [71,72]). Consequently, the relativistic approach explains, at the same time, the depth of the average potential (around $50 \mathrm{MeV}$ ), the magnitude of the spin-orbit term, and the small pseudo-spin-orbit splitting. In this context, it should be noted that the traditional picture of nuclear shells and magic gaps proposed fifty years ago [73,74] should not be taken for granted. As discussed in Sec. 7 below, modifications of shell structure are expected in the limit of a large $N / Z$ ratio.

We have learned a great deal about modes of nuclear excitations using phenomenological models, often based on ingenious intuition and symmetry considerations. These models, approaches, and approximations have been extremely successful in interpreting nuclear states and classifying nuclear states and decays. Based on this experience, and thanks to developments in theoretical modeling and computer technology, we are now on the edge of the microscopic description. 
By taking advantage of modern many-body algorithms, one can now shorten the cycle theory $\leftrightarrow$ experiment $\leftrightarrow$ theory. Most many-body methods of theoretical nuclear structure were introduced a long time ago (in the fifties and sixties). However, thanks to incredible progress in hardware and numerical techniques, it is only now that we can use these methods in their full glory.

Figure 2, bottom, includes a schematic illustration of the hierarchy of theoretical models spanning the chart of the nuclides. By exploring connections between these models, nuclear theory aims to develop a unified description of the nucleus. It probably would be very naive to think of the behavior of a heavy nucleus directly in terms of the underlying quark-gluon dynamics, but undoubtedly the understanding of the bridges in Fig. 1 will make this goal qualitatively possible.

\section{Nuclear Modes and Their Time Scales}

Considering that the atomic nucleus is, in a good approximation, a cluster of strongly interacting nucleons (or pairs of nucleons) wandering solo in all directions, the very existence of nuclear collective motions such as rotations or vibrations, with all particles moving in unison, is rather astonishing.

When discussing nuclear collective motion, such as rotations and vibrations, one is often making analogies to molecules and their collective modes. As a matter of fact, many elements of the unified model have been directly transferred to nuclear physics from molecular physics [75]. But there is a fundamental difference between the nuclear and molecular world: in molecules, the fast electronic motion is strongly coupled to the equilibrium position of slowly moving ions.

This point is nicely illustrated in Fig. 9, which shows the measured spectrum of a diatomic molecule $\mathrm{N}_{2}$. Each electronic excitation represents a bandhead upon which vibrational and rotational states are built. Vibrational states are indicated by a vibrational quantum number $\nu(\nu=0$ representing the zero-phonon state, $\nu=1$ is a one-phonon state, and so on). Rotational levels are not plotted - there are far too many to be displayed. The time scale of molecular modes is governed by the hierarchy of excitation energies. The electronic excitations are of the order of $10^{5} \mathrm{~cm}^{-1}$ (which in "nuclear" units corresponds to an $\mathrm{eV}$ ), vibrational frequencies are $\sim 10^{3} \mathrm{~cm}^{-1}$, and rotational energies are $\sim 10^{-1} \mathrm{~cm}^{-1}$. This means that the single-particle electronic motion is from two to six orders of magnitude faster than molecular collective modes. Consequently, the adiabatic assumption, based on the separation of all molecular degrees of freedom into fast and slow ones, is justified due to different time scales.

Unfortunately, the total $A$-body wave function of the nucleus cannot, in general, be expressed in terms of slow and fast components. This is because (i) the collective nuclear coordinates are auxiliary variables which depend, in a complex way, on fast nucleonic degrees of freedom, and (ii) the nuclear residual interactions are not small. How good is the time separation between single-particle and collective nuclear motion? The typical single-particle period (i.e., the average time it takes a neutron or a proton to go across the nucleus), $T_{\mathrm{s} \text {. }}=4 R / v_{\mathrm{F}}$ 


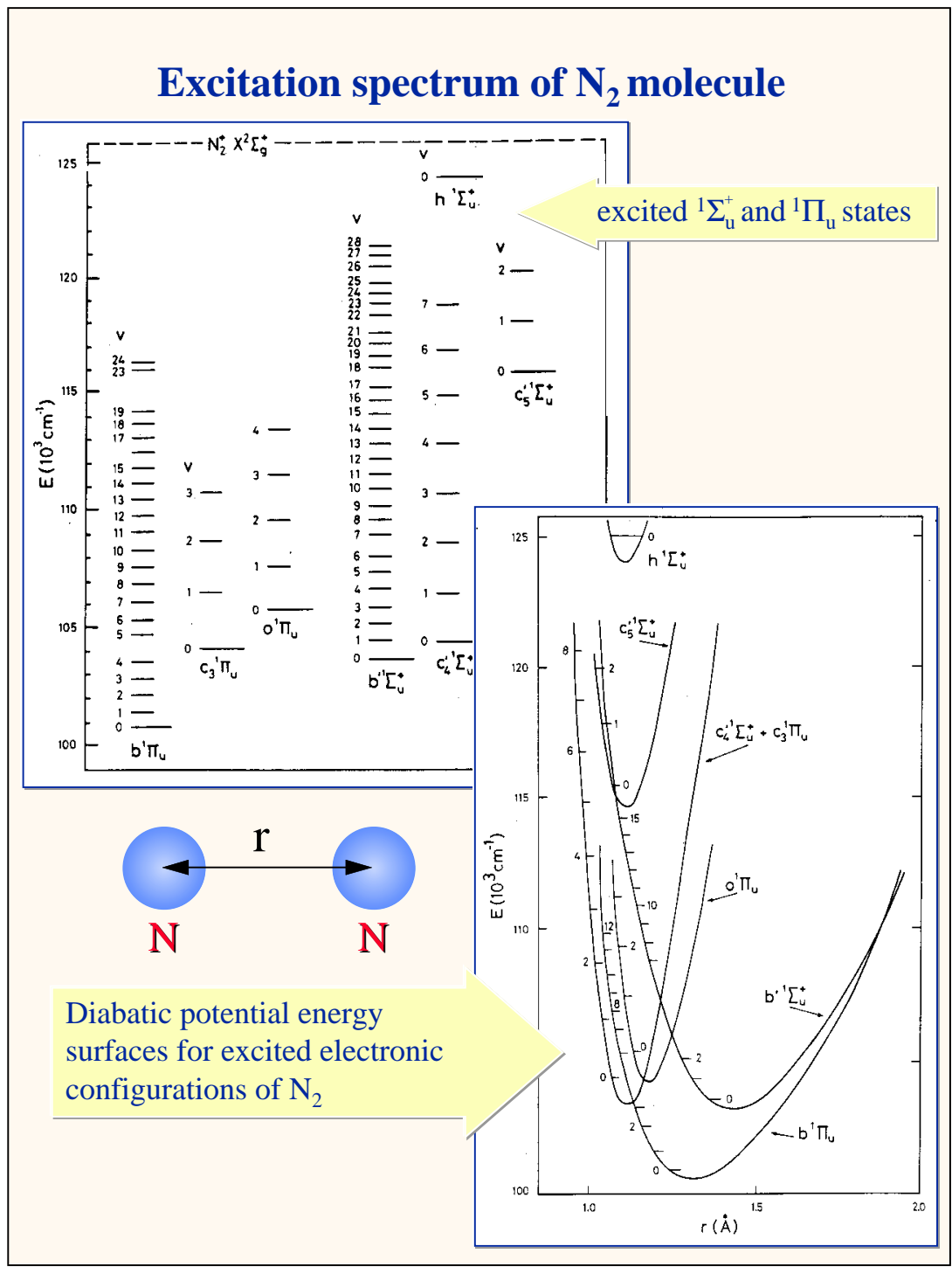

Fig. 9. Top: vibrational spectrum of $\mathrm{N}_{2}$ molecule. Bottom: collective potentials, corresponding to different electronic configurations, as functions of the internuclear radius $r$ (from Ref. [76]).

[where $R$ is the nuclear radius and $v_{\mathrm{F}}$ is the Fermi velocity $(\sim 0.29 c)$ ], is approximately $3 \cdot 10^{-22}$ sec. (The unit $10^{-22} \mathrm{sec}$ is sometimes referred to as babysecond. The single-particle time scale is of the order of several babysec.) The typical period of nuclear rotation $\left(T_{\text {rot }} \approx 10^{-21} \mathrm{sec}\right)$ is only $\sim 30$ times greater than $T_{\text {s.p. }}$, and for nuclear vibrations the period of oscillations is only slightly greater than the 
single-particle period. It is truly amazing that these relatively small differences in time scales seem to be sufficient to create rotating or vibrating potentials, common for all nucleons!

How "good" are the actual nuclear rotations and vibrations? Let us consider some representative experimental examples. Probably the most spectacular "molecular" rotational-vibrational nuclear spectrum is that of ${ }^{240} \mathrm{Pu}$ in its superdeformed minimum [77] (Fig. 10). This beautiful and rich structure, observed in a ${ }^{238} \mathrm{U}(\alpha, 2 \mathrm{n})$ reaction, shows one- and two-phonon deformed quadrupole and octupole vibrational states, as well as the rotational bands built upon them. Its spectrum shows some similarity with the molecular pattern; several low-lying excitations can be associated with one-phonon states, and some of its levels are believed to have a two-phonon nature. At first sight, the collective structures seen in ${ }^{240} \mathrm{Pu}$ are fairly regular. However, after closer inspection, many deviations from the perfect rotational and vibrational pattern can be seen. For example, the moments of inertia of rotational bands are by no means constant but show local variations as a function of angular momentum. Also, the positions of vibrational bandheads differ from the harmonic limit. Such deviations indicate that the nuclear motion is not completely collective, i.e., that the collective modes result from coherent superpositions of single-particle nucleonic excitations.

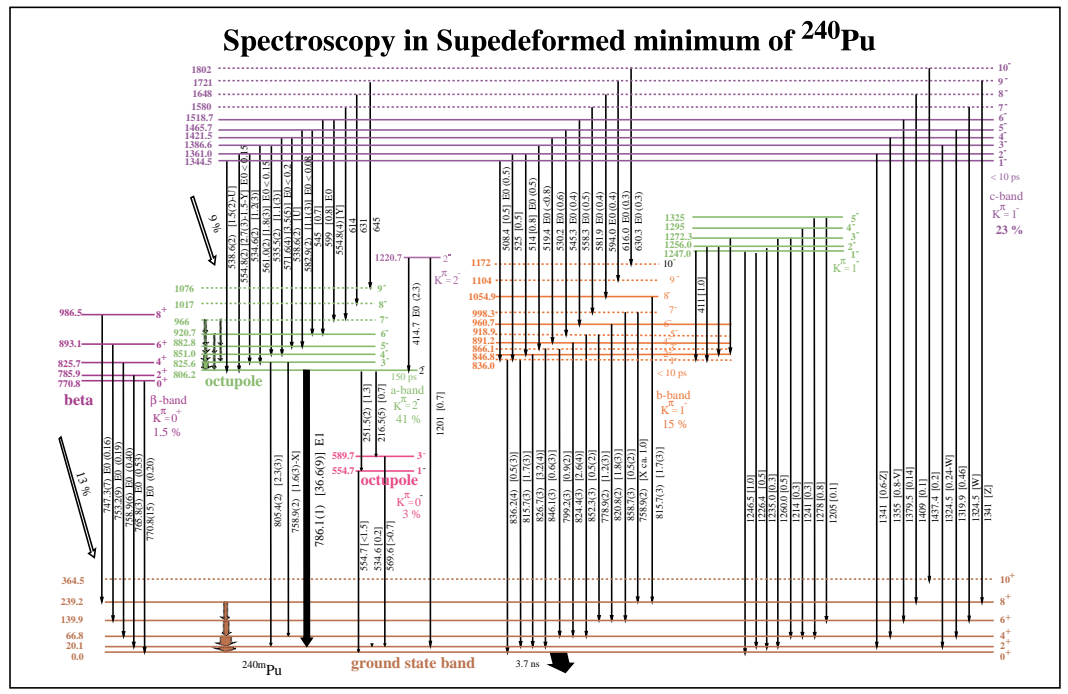

Fig. 10. Excitation spectrum of ${ }^{240} \mathrm{Pu}$ in the superdeformed minimum. Low-lying states have characteristic rotational-vibrational structure. (From Ref. [77].)

Another extreme case is shown in Fig. 11, which displays the excitation spectrum of ${ }^{148} \mathrm{Gd}$. This spectrum looks very irregular; it is characteristic of manyparticle many-hole excitations in an almost spherical nucleus. There are many 


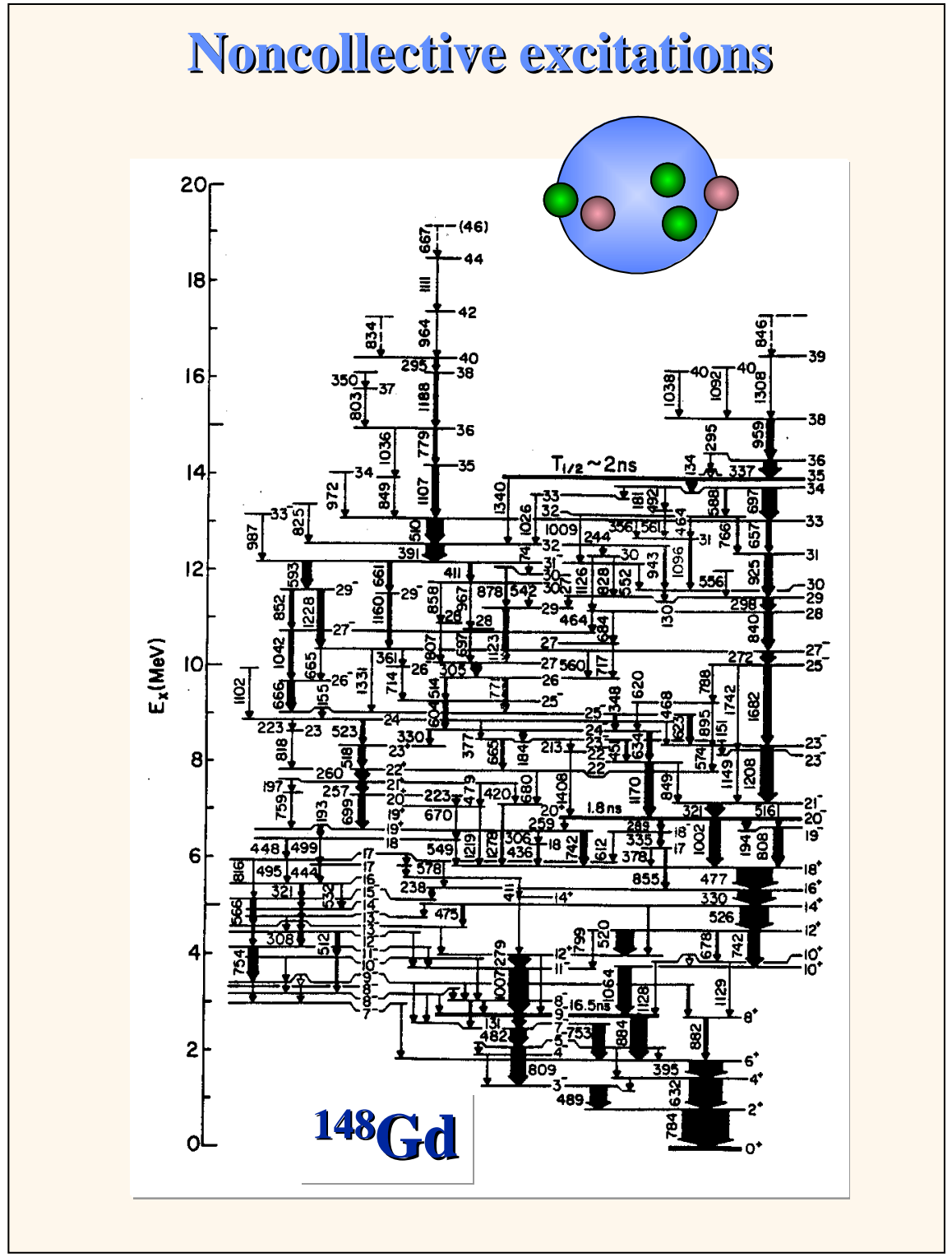

Fig. 11. Excitation spectrum of ${ }^{148} \mathrm{Gd}$. It is a beautiful example of noncollecive nucleonic motion (from Ref. [78]).

states of different angular momentum and parity connected via relatively weak electromagnetic transitions. In spite of the fact that the excitation pattern looks "chaotic", most of the noncollective states of ${ }^{148} \mathrm{Gd}$ can be beautifully described in terms of well-defined quantum numbers of the nuclear shell model [79]. The 
collective spectrum of ${ }^{240} \mathrm{Pu}$ discussed above was not perfectly collective. Likewise, in the "mess" of noncollective levels in ${ }^{148} \mathrm{Gd}$ one can find some elements of collectivity. For instance, the energies of the four lowest states $0^{+}, 2^{+}, 4^{+}$, $6^{+}$, when plotted as functions of angular momentum, exhibit a characteristic parabolic pattern. As was realized around forty years ago [80], such a sequence of states indicates the presence of pairing - a collective phenomenon.

The next example illustrates the coexistence effect. It is a particular case of the large amplitude collective motion where several nuclear configurations, characterized by different intrinsic properties, compete [83]. In the spectrum of

${ }^{152}$ Dy, shown in Fig. 12, one can recognize the noncollective structure resembling that in ${ }^{148} \mathrm{Gd}$, a collective rotational band (interpreted in terms of a deformed triaxial configuration), and super-collective superdeformed bands corresponding to very large shape-elongations which are structurally isolated from the rest of the spectrum.

The last experimental example shows yet another case of nuclear collectivity - high-frequency nuclear vibrations. Figure 13 displays the cross section for the scattering of the relativistic ${ }^{208} \mathrm{~Pb}$ on a xenon nucleus. The first maximum in the cross section, which appears at $\sim 14 \mathrm{MeV}$, can be associated with the one-phonon giant dipole resonance (i.e., very fast vibration of protons against neutrons). It has been suggested [84] that the local maximum at an energy of $\sim 28 \mathrm{MeV}$ is a two-phonon giant dipole vibration. In the data shown in Fig. 13 one can also find isoscalar and isovector giant quadrupole resonances. The giant nuclear excitations are always strongly fragmented; their widths are usually more than 5 $\mathrm{MeV}$. It is worth noting that the time scale corresponding to giant two-phonon vibrations is shorter than 1 babysec! The unusual harmonicity of this mode is not understood well.

One of the outstanding challenges in nuclear structure is to understand the mechanism governing the nature of nuclear collective excitations. By studying nuclear rotations and vibrations, one is probing the details of the nuclear force in a strongly interacting medium.

\section{Nuclear Deformations}

The phenomenon of nuclear deformation has a long and interesting history. As early as 1924 it was suggested by Pauli [85] that the hyperfine structure of atomic and molecular energy levels resulted from the electromagnetic interaction with nonspherical atomic nuclei. (The experimental evidence was given ten years later by Schüler and Schmidt [86].) The fact that nuclei need not be spherical was then emphasized by N. Bohr in his classic paper on the nuclear liquid-drop model [87] in which he introduced the concept of nuclear shape vibrations. If a quantummechanical system is deformed, its spatial density is anisotropic. For a deformed system it is possible to define its orientation as a whole and this naturally leads to the presence of collective rotational modes. This possibility was realized as early as 1937 by N. Bohr and Kalckar [88] who had estimated for the first time the energies of lowest rotational excitations and introduced the notion of the 


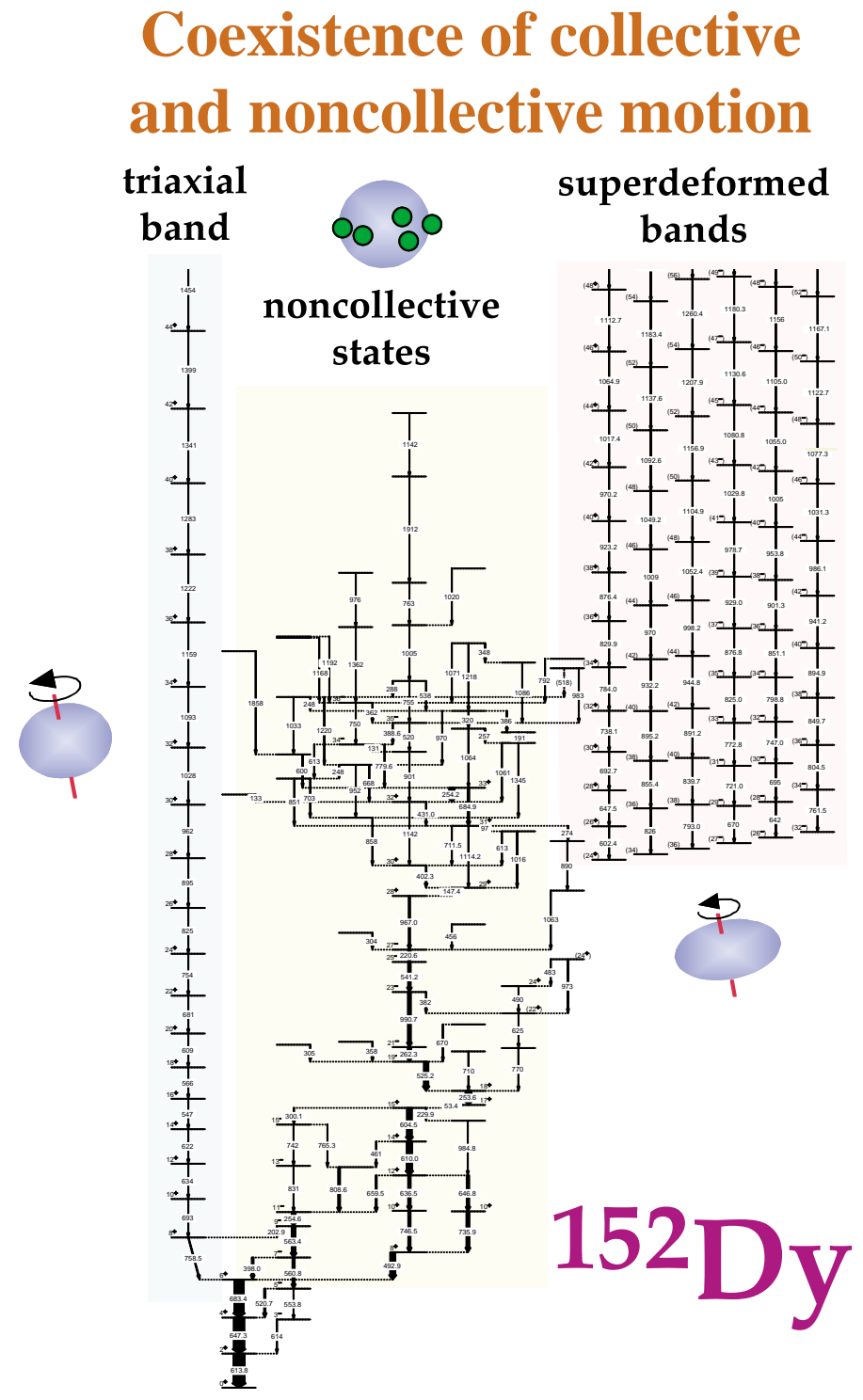

Fig. 12. Excitation spectrum of ${ }^{152}$ Dy. It is a spectacular example of the coexistence of collective and noncollecive nucleonic modes. (Experimental data were taken from Refs. [81,82].)

nuclear moment of inertia (see also work by Teller and Wheeler [89]). In 1939, Meitner and Frisch [90] and N. Bohr and Wheeler [91] stressed the role of shape degrees of freedom during the fission process. 


\section{High-frequency nuclear vibrations (giant resonances)}
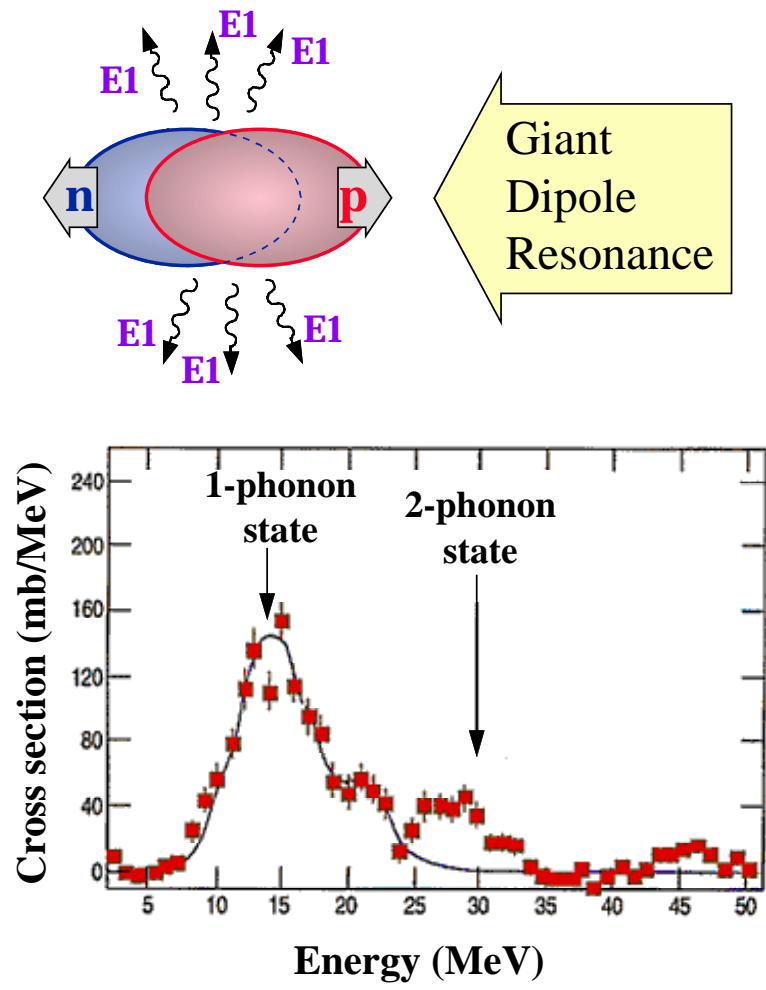

Fig. 13. One-phonon and two-phonon giant resonances in ${ }^{208} \mathrm{~Pb}$. (Experimental data were taken from Ref. [84].)

In 1950, Rainwater [92] observed that the experimentally measured large quadrupole moments of nuclei could be explained in terms of the deformed shell model; i.e., the extension of the spherical shell model to the case of the deformed average potential. In this picture, the deformed field was a direct consequence of single-particle motion in anisotropic orbits. In a following paper [93], A. Bohr formulated the basis of the particle-rotor model, introduced the concept of the intrinsic (body-fixed) nuclear system defined by means of shape deformations, and regarded nuclear shape and orientation as dynamical variables (in this connection, see also Refs. [94-98]).

The basic microscopic mechanism leading to the existence of nuclear deformations was proposed by A. Bohr in his paper on "The Coupling of Nuclear Surface Oscillations to the Motion of Individual Nucleons" [99]. As a matter of 
fact, this title is probably the most precise (and shortest) answer to the question: Why are nuclei deformed? The interaction between the single-particle motion of valence nucleons and the collective excitations (multipole vibrations) of the core was represented by Bohr by the particle-vibration coupling Hamiltonian,

$$
H_{\mathrm{PV}}=-\sum_{\lambda \mu} \kappa_{\lambda \mu} \alpha_{\lambda \mu} \sum_{i} f\left(r_{i}\right) Y_{\lambda \mu}\left(\Omega_{i}\right)
$$

where $\alpha_{\lambda \mu}$ is the amplitude of the nuclear deformation of the core, $f\left(r_{i}\right) Y_{\lambda \mu}\left(\Omega_{i}\right)$ is the multipole moment of the $i$-th valence nucleon, and $\kappa_{\lambda \mu}$ stands for the particle-vibration coupling strength. The coupling (1) is a central element in the analysis of nuclear collective modes and nuclear deformations.

It was forty years ago when the theory of the coupling between nuclear singleparticle and collective degrees of freedom was presented by A. Bohr and Mottelson [100] and Hill and Wheeler [101]. These two works form the intellectual and conceptual basis of the nuclear unified model that turned out to be one of the most powerful tools of nuclear structure. Since the main objective of this section is to discuss the microscopic aspects of nuclear deformation rather than to talk about the past, I should stop these brief historical remarks here. But before doing so, let me mention that already in 1935, in London, Jahn and Teller demonstrated that the electronic degeneracy in molecules could destroy the symmetry on which it was based [102]. As discussed below (see also Refs. [103,104]), a similar mechanism is responsible for the presence of deformed nuclear shapes.

\section{$5.1 \quad$ Nuclear Jahn-Teller Effect}

The Jahn-Teller effect, or the phenomenon of spontaneous symmetry-breaking, first proposed for molecules, turned out to appear in many fields of physics. Deformation is a common phenomenon that appears in mesoscopic systems with many degrees of freedom (atomic nuclei, atomic clusters). It also appears in field theory (Higgs mechanism), in physics of superconductors (Meissner effect), in condensed matter physics (numerous phenomena related to spin resonance and relaxation), and other fields of physics. Since the microscopic origin of the JT effect is not well known in the nuclear structure community, this section contains a slightly higher level of detail as compared to other sections.

The adiabatic approximation by Born and Oppenheimer [105] is the natural starting point in the discussion of spontaneous symmetry-breaking. It is based on the assumption that system coordinates can be separated into slow (collective, relevant) coordinates, $\{\boldsymbol{Q}\}=\left\{Q_{j}\right\}$, and fast (noncollective, irrelevant) coordinates, $\{\boldsymbol{x}\}=\left\{x_{i}\right\}$. The separation obviously depends on the actual physical problem.

The Hamiltonian for the whole system can be written as

$$
\hat{H}=T_{\boldsymbol{Q}}+T_{\boldsymbol{x}}+V(\boldsymbol{Q}, \boldsymbol{x}),
$$

where $T_{\boldsymbol{Q}}$ and $T_{\boldsymbol{x}}$ are kinetic energies associated with slow and fast coordinates, respectively. At this point the slow and fast coordinates are treated on the same 
footing. The equations of motion for the Hamiltonian (2) can be written in a canonical form and the motion of the system can be described in the full phase space of collective and non-collective coordinates and associated momenta.

The stationary Schrödinger equation with Hamiltonian (2) is solved in two steps. First, at a given point in the collective phase space one solves the eigenproblem for the non-collective Hamiltonian $h=T_{\boldsymbol{x}}+V(\boldsymbol{Q}, \boldsymbol{x})$ :

$$
\left[T_{\boldsymbol{x}}+V(\boldsymbol{Q}, \boldsymbol{x})\right] \Psi_{n}(\boldsymbol{x} ; \boldsymbol{Q})=E_{n}(\boldsymbol{Q}) \Psi_{n}(\boldsymbol{x} ; \boldsymbol{Q})
$$

where $\Psi_{n}(\boldsymbol{x} ; \boldsymbol{Q})$ is the fast (single-particle) wave function which contains the slow coordinates $\{\boldsymbol{Q}\}$ s parametrically, and $E_{n}(\boldsymbol{Q})$ s are the single-particle energies at the point $\{\boldsymbol{Q}\}$. Consequently, the $E_{n}(\boldsymbol{Q})$ s are the static potential energy surfaces in the collective space.

In the second step, the total wave function of the system is written as a product

$$
\Psi=\sum_{n} \Psi_{n}(\boldsymbol{x} ; \boldsymbol{Q}) \chi_{n}(\boldsymbol{Q})
$$

The collective wave functions $\chi_{n}(\boldsymbol{Q})$ arise from the effective potential appropriate to each single-particle state. By combining Eqs. (2-4), one obtains the coupled-channel Schrödinger equation for $\chi_{n}(\boldsymbol{Q})$ :

$$
\sum_{m}\left[\delta_{m n} T_{\boldsymbol{Q}}+V_{m n}(\boldsymbol{Q})\right] \chi_{m}(\boldsymbol{Q})=E \chi_{n}(\boldsymbol{Q})
$$

The effective collective potential $V_{m n}(\boldsymbol{Q})$ takes into account the collective response of the single-particle energy on collective coordinates $\{\boldsymbol{Q}\}$. It contains the coupling term between different single-particle states $n$ and $m$, defined through

$$
\sum_{m}\langle n| T_{\boldsymbol{Q}}\left\{|m\rangle \chi_{m}(\boldsymbol{Q})\right\}
$$

If the coupling term is weak, the eigenfunctions of the total system are just products of collective and single-particle wave functions.

In molecular physics, the electronic states (described by means of fast coordinates) are strongly coupled to the equilibrium position of the ions (described by means of slow coordinates). The adiabatic assumption is justified due to different time scales of nuclear and electronic motions (see Fig. 9 and related discussion). In nuclear physics, the $\{\boldsymbol{Q}\}$ s are fields characterizing various collective modes and $\{\boldsymbol{x}\}$ s are remaining coordinates. This separation is by no means simple; the choice of proper nuclear collective coordinates is a long-standing problem $[28,106]$. Indeed, as discussed early in Ref. [93]: "In contrast with the molecular case, there are here no heavy particles to provide the necessary rigidity of the structure. However, nuclear matter appears to have some of the properties of coherent matter which makes it capable of types of motion for which the effective mass is large as compared with the mass of a single nucleon." The variety and richness of nuclear collective modes, appearing even in very light nuclei, suggest that the adiabatic approximation is, very often, a powerful concept. 


\subsection{Level Crossing}

In many cases the off-diagonal matrix element in Eq. (6) does not vanish and non-collective modes are strongly coupled through the collective field. An extreme coupling occurs when there are two or more non-collective states which are degenerate (or almost degenerate) at some point $\{\boldsymbol{Q}\}=\left\{\boldsymbol{Q}_{c}\right\}$, i.e.,

$$
E_{k}\left(\boldsymbol{Q}_{c}\right) \approx E_{l}\left(\boldsymbol{Q}_{c}\right) \quad(k \neq l) .
$$

The matrix of the collective momentum $\partial / \partial \boldsymbol{Q}$, a Berry vector

$$
(\boldsymbol{A})_{l k}=i\left\langle l\left|\frac{\partial}{\partial \boldsymbol{Q}}\right| k\right\rangle,
$$

is the single most important quantity to determine the validity of the adiabatic approximation. For small collective velocities and $\boldsymbol{A}$ slowly changing in time, the motion of the system is adiabatic and can be well described by means of the perturbation theory. Sometimes, off-diagonal matrix elements of $(\boldsymbol{A})_{l k}$ disappear (e.g., for symmetry reasons), and Eq. (6) contains only diagonal matrix elements $(\boldsymbol{A}) l$ which can be eliminated by means of a simple gauge transformation.

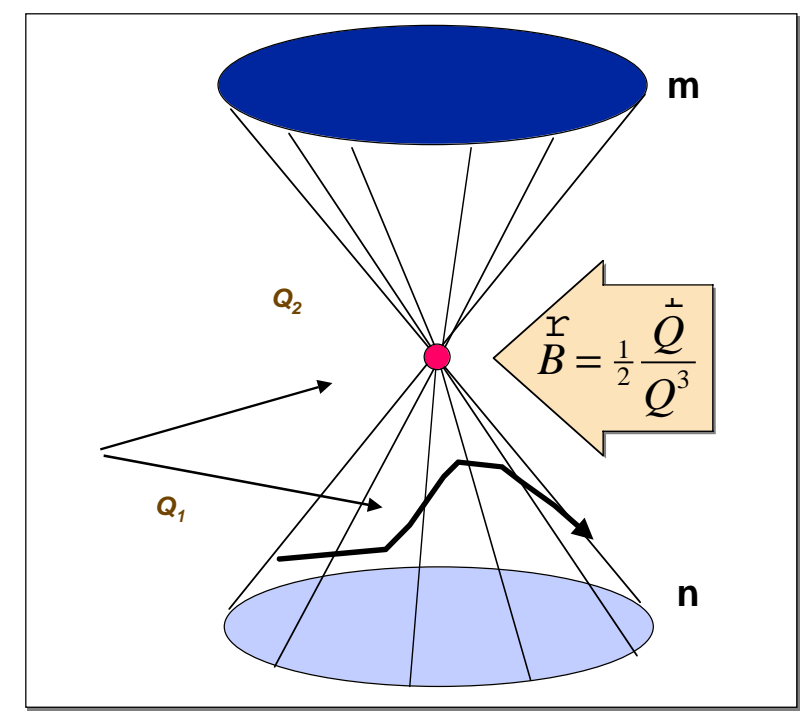

Fig. 14. Conical intersection between two potential energy surfaces $m$ and $n$ in a collective $\{\boldsymbol{Q}\}$-space. The crossing point contains a generalized monopole potential that tries to repel the system from the point of degeneracy. (Based on Ref. [107].)

A wealth of very interesting physics appears at the point of degeneracy, $\{\boldsymbol{Q}\}=\left\{\boldsymbol{Q}_{c}\right\}$. Unlike in the static situation, it is now impossible to eliminate (8) through the gauge transformation. In particular, the Berry phase on a closed 
path in $\{\boldsymbol{Q}\}$-space does not vanish if the path encloses the point of degeneracy. Geometrically, the crossing point can be viewed as a diabolical point containing a generalized vector potential [107-109] singular at $\boldsymbol{Q}_{c}$, see Fig. 14. Consequently, during its collective motion the system will always try to go as far as possible from the point of degeneracy, analogously to the motion of a charged particle in the magnetic field of a monopole[108,110]. In the vicinity of $Q_{c}$, the actual eigenstates of $\hat{H}$ are mixtures of diabatic states. Consequently, around the crossing point (i) the original (fast) quantum numbers are gone and the description in terms of intrinsic Hamiltonian breaks down, (ii) the motion of the system cannot be separated into the collective and non-collective parts, and (iii) the notion of the collective adiabatic potential loses its meaning[111].

\subsection{Vibronic Coupling}

Let us assume that collective normal modes (e.g., nuclear vibrational modes) transform according to the irreducible representations $\mathcal{T}(\lambda)$ of the symmetry group $\mathcal{T}$. Therefore, they can be written as $\left\{Q_{\lambda \mu}\right\}$, where $\mu$ labels the component of the representation $\lambda$. The single-particle states belong to a certain group representation $\mathcal{T}(J)$ of dimension $[J]$, i.e., $|n, \boldsymbol{x}\rangle=|J M, \boldsymbol{x}\rangle$ ( $M$ labels the component of the representation $J)$. In order to analyze the particle-vibration coupling, it is convenient to expand the vibronic potential $V_{m n}$ defined in Eq. (5) with respect to collective coordinates around the point of degeneracy, $\left\{\boldsymbol{Q}_{c}\right\}=\{0\}$ :

$$
V_{J M, J M^{\prime}}(\boldsymbol{Q})=V_{J M, J M^{\prime}}(0)+\sum_{\lambda \mu} Q_{\lambda \mu}\left(\frac{\partial V_{J M, J M^{\prime}}}{\partial Q_{\lambda \mu}}\right)_{(0)}+\cdots
$$

Then by the Hellmann-Feynman theorem and the Wigner-Eckart theorem, the linear vibronic coupling term (often referred to as the JT matrix element) can be written as

$$
\left(H_{\mathrm{JT}}\right)_{M M^{\prime}}=[J]^{-\frac{1}{2}} \sum_{\lambda \mu} Q_{\lambda \mu}\left\langle J\left\|\left(\frac{\partial H}{\partial Q_{\lambda}}\right)_{(0)}\right\| J\right\rangle\left\langle J M^{\prime} \lambda \mu \mid J M\right\rangle,
$$

where $\langle J\|\ldots\| J\rangle$ is the reduced matrix element, and $\left\langle J M^{\prime} \lambda \mu \mid J M\right\rangle$ is the corresponding Clebsch-Gordan coefficient for representations $\lambda$ and $J$.

It is seen immediately from Eq. (10) that the JT matrix element is zero if the representation $\mathcal{T}(\lambda)$ is not contained in the product $\mathcal{T}(J) \otimes \mathcal{T}(J)$. This would mean that in such a situation the collective mode $\lambda \mu$ does not couple degenerate single-particle states. The diagonal constant of the linear coupling has the sense of the force with which the single-particle part of the system in state $|J M, \boldsymbol{x}\rangle$ affects the collective part in the direction of the collective coordinate $Q_{\lambda}$.

The vibronic coupling between single-particle nucleonic motion and collective modes can be estimated by means of a weak coupling model [99,112]. Here the collective subspace refers to the vibrational modes of the core, while the fast subspace is associated with the single-particle coordinates of valence particles. 
The particle-core interaction, which can be directly derived from the multipolemultipole force, is given by Eq. (1), i.e.,

$$
V(\boldsymbol{Q}, \boldsymbol{x})=-\sum_{\lambda \mu} \kappa_{\lambda \mu} Q_{\lambda \mu} q_{\lambda \mu}^{v a l}
$$

The symmetry group of interest is the group of rotations characterized by representations $(J, M)$. Consequently, the JT matrix element becomes

$$
\left(H_{\mathrm{JT}}\right)_{M^{\prime} M^{\prime \prime}}=-(2 J+1)^{-\frac{1}{2}} \sum_{\lambda \mu} \kappa_{\lambda \mu} Q_{\lambda \mu}\left\langle J\left\|q_{\lambda}^{v a l}\right\| J\right\rangle\left\langle J M^{\prime \prime} \lambda \mu \mid J M^{\prime}\right\rangle .
$$

It is seen from Eq. (12) that the high- $j$ states contribute the most to the JT matrix element. Indeed, for those states the value of $\left\langle J\left\|q_{\lambda}^{v a l}\right\| J\right\rangle$ is largest.

A " $Q \cdot q$ " coupling (11), where $Q$ represents the collective (bosonic) field and $q$ describes the single-particle (fermionic) subsystem, is well known in many fields of physics (see, e.g., Ref. [113]). Examples of $(Q, q)$ are: (photons, charged particles), (mesons, nuclei), (phonons, electrons), and (phonons, nuclei). In all of these cases, the potential energy $V(\boldsymbol{Q}, \boldsymbol{x})$ in (2) can be approximated by

$$
V(\boldsymbol{Q}, \boldsymbol{x})=V(\boldsymbol{Q})+\kappa \boldsymbol{Q} \cdot \boldsymbol{q}(\boldsymbol{x})+h(\boldsymbol{x}) .
$$

Consequently, the physics of the particle-vibration (fermion-boson) coupling is rather similar for all the systems mentioned above.

\subsection{Static Nuclear Deformations}

Let us illustrate the nuclear JT effect using simple arguments based on the Random Phase Approximation. A simple separable spherical nuclear Hamiltonian representing vibrations associated with the operator $\hat{Q}$ can be written as:

$$
H=\sum_{i} e_{i} c_{i}^{+} c_{i}-\frac{1}{2} \kappa \hat{Q}^{+} \cdot \hat{Q} .
$$

This Hamiltonian contains two basic ingredients of nuclear shape-collectivity, namely (i) the single-particle term representing the individual motion of nucleons around the Fermi surface, and (ii) the multipole vibrational field $Q$ that generates the collective motion.

Within the formalism of RPA [28], the excitations of the system, $\omega_{\mathrm{RPA}}$, are solutions of the so-called dispersion equation:

$$
R\left(\omega_{\mathrm{RPA}}\right)=\sum_{p h} \frac{2|\langle p h|Q| 0\rangle|^{2} \epsilon_{p h}}{\epsilon_{p h}^{2}-\omega_{\mathrm{RPA}}^{2}}=\frac{1}{\kappa}
$$

where $R\left(\omega_{\mathrm{RPA}}\right)$ is the dispersion function and $\epsilon_{p h}$ stands for the energy of a particle-hole excitation, $\epsilon_{p h}=e_{p}-e_{h}$. The lowest root of Eq. (15) usually represents the low-frequency collective vibrational state. 


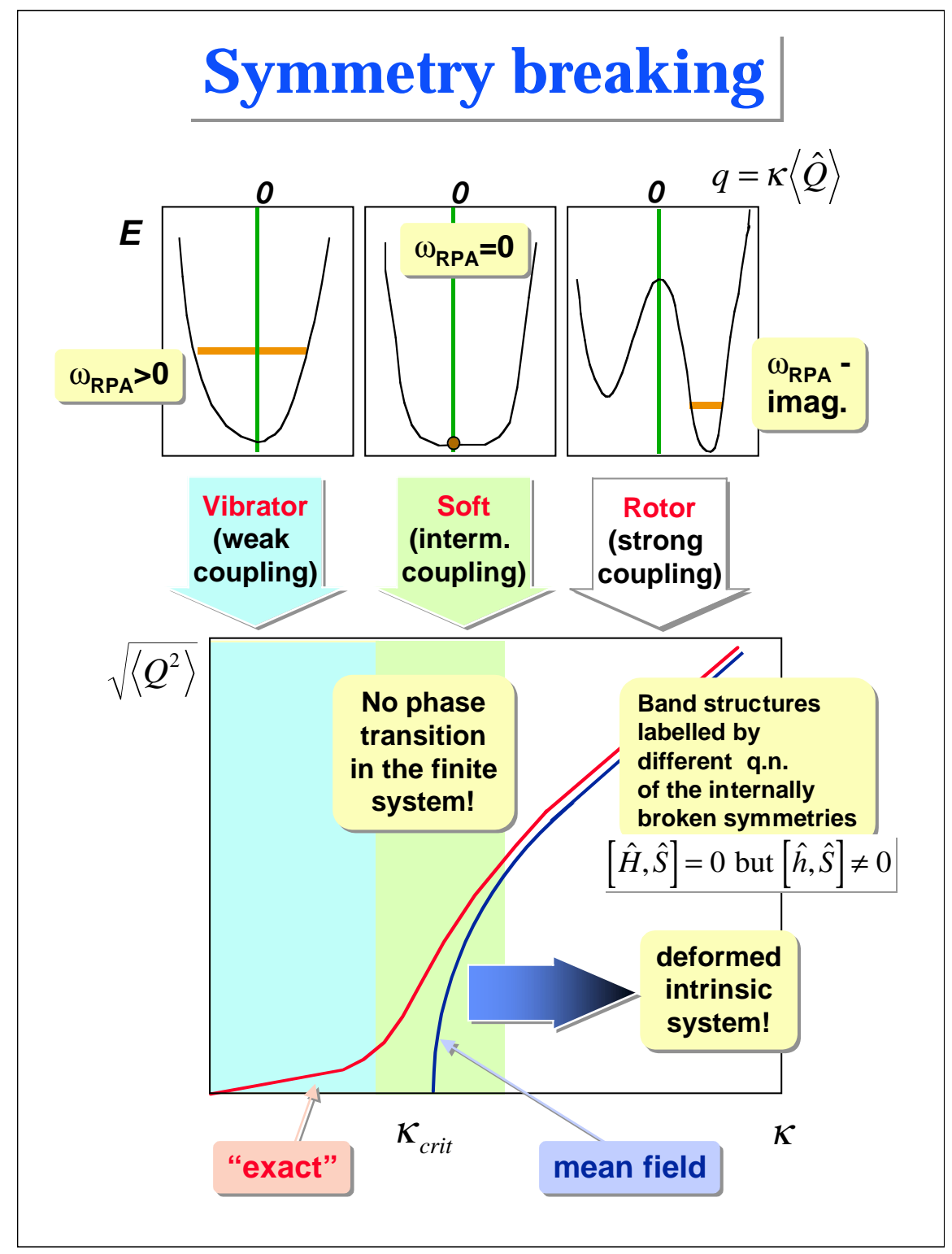

Fig. 15. Schematic diagram illustrating the mechanism of static nuclear deformation in the the mean-field picture. See text for details.

The total HF energy is shown as a function of average self-consistent deformation (collective coordinate) $q=\kappa\langle\hat{Q}\rangle_{H F}$ in the top portion of Fig. 15. Let us discuss some properties of the RPA solutions corresponding to different values of coupling constant $\kappa$. These three cases are represented in Fig. 15, top portion. If 
the coupling constant $\kappa$ is small (weak coupling or vibrational limit), the lowestenergy solution is depressed with respect to the lowest non-collective excitation, and the wave function of the collective state becomes a coherent superposition of many particle-hole configurations. In this situation, characteristic of harmonic vibrations, the potential energy curve is quadratic around the equilibrium point, $q=0$, and the stiffness

$$
C=\left(d^{2} E / d q^{2}\right)_{q=0}
$$

is greater than zero. In this case, $\omega_{\mathrm{RPA}}$ represents the energy of the vibrational one-phonon excitation.

If the value of $\kappa$ is increased, the energy surface softens, the low-energy vibration becomes more collective, and the vibrational frequency decreases. At the critical point (transition from spherical to deformed) when $\kappa$ becomes equal to $\kappa_{\text {crit }}=1 / R(0)$, the lowest RPA solution appears at zero energy, i.e., the collective state becomes degenerate with the ground state. (This is precisely the famous Goldstone boson!) At this point of a boson condensation, the stiffness $C$ vanishes and the harmonic approximation breaks down. Since the system cannot have two ground states, it becomes unstable against vibration induced by the multipole-multipole force [114].

A further increase of the coupling constant leads to permanent shape deformation. At this limit (strong coupling), the lowest solution of Eq. (15) has imaginary energy. Here, the stiffness at a spherical shape becomes negative and the energy becomes minimal at some non-zero value of $q=q_{0} \neq 0$. The energy of the lowest collective vibrational state built upon the deformed solution is schematically indicated in Fig. 15. It can be computed by means of RPA equations analogous to those of Eq. (15), but pertaining to the deformed minimum. However, the lowest excitations of the nucleus in this limit are not vibrations but rotations. The presence of stable deformations makes it possible to distinguish between various orientations of the nucleus in space; hence rotational bands (i.e.,collective excitations connected by enhanced transition matrix elements) appear.

The lower portion of Fig. 15 presents schematically the $q$-vs. $\kappa$ "phase diagram". Within the static mean-field theory, which becomes exact at infinite particle number, the self-consistent deformation is zero below the critical point, at which the first order phase transition takes place. For $\kappa>\kappa_{\text {crit }}$ the system has static deformation (shape deformation, pair deformation, etc.). In this context, it should be emphasized that the deformed picture is valid for rather large values of coupling strength, or for very large particle numbers (for instance, in solid state physics, where $\mathrm{N} \sim 10^{23}$ ). Atomic nuclei are relatively small systems and the finite size effects, which manifest through dynamical correlations (fluctuations), are extremely important. The fluctuations wash out the transition from the "spherical" to "deformed" phase, and result in a smooth and continuous pattern of $q$, as indicated schematically in Fig. 15.

In the rotational limit, one can define the intrinsic system of the nucleus. Although the nuclear (laboratory-system) Hamiltonian does commute with the symmetry broken by the operator $\hat{Q}$, the intrinsic-system one-body Hamiltonian (mean-field Hamiltonian, HF Hamiltonian) does not. This is precisely the essence 
of the symmetry-breaking: rotational bands are a manifestation of internally broken symmetries. Nuclear states forming a collective band are labeled by quantum numbers of these broken symmetries. Examples are rotational bands in nuclei having quadrupole deformations (here the collective operator is the quadrupole moment, the broken symmetry is rotational invariance, and rotational bands are labeled by angular momentum quantum numbers), octupole deformations (here the collective operator is the octupole moment, the broken symmetry is space inversion, and rotational bands are parity doublets), and pairing correlations (here the collective operator is the pair operator, the broken symmetry is particle number, and rotational bands are the ground states of neighboring even-even nuclei). See Sec. 6.1 for more examples.

Based on the above discussion, one can make an interesting observation: if the lowest particle-hole energy approaches zero, the lowest pole of the dispersion function (Eq. (15)) becomes so small that the lowest solution is always degenerate with the ground state, independent of the magnitude of the coupling strength. Consequently, if the Fermi level lies just between two or more (almost) degenerate states, the system should be unstable with respect to the mode that couples these states. This is precisely the JT mechanism.

If pairing correlations are present, the dispersion relation given by Eq. (15) is slightly modified and, in the BCS approximation, it becomes:

$$
\sum_{\alpha, \alpha^{\prime}} \frac{2\left|\left\langle\alpha^{\prime}|Q| \alpha\right\rangle\right|^{2}\left(u_{\alpha} v_{\alpha^{\prime}}+u_{\alpha^{\prime}} v_{\alpha}\right)^{2}\left(E_{\alpha}+E_{\alpha^{\prime}}\right)}{\left(E_{\alpha}+E_{\alpha^{\prime}}\right)^{2}-\omega_{\mathrm{RPA}}^{2}}=\frac{1}{\kappa}
$$

where $E_{\alpha}=\sqrt{\left(e_{\alpha}-e_{F}\right)^{2}+\Delta^{2}}$ are the quasiparticle energies, and $u_{\alpha}$ and $v_{\alpha}$ are the usual BCS occupation coefficients. The main effect of pairing correlations is the change in the energy denominator in Eq. (17), which now is much larger than the particle-hole energies in Eq. (15). Indeed, the energy of the lowest pole becomes $2 \Delta \simeq 2 \mathrm{MeV}$. Together with the reduction of the numerator of Eq. (17) through the $u v$ factor, pairing correlations tend to increase the critical value of $\kappa$. One can thus say that pairing has a tendency to make the system more spherical. The extreme JT effect, with very weak residual interaction, can take place in excited nuclear states. For instance, at high angular momentum the number of broken nucleonic pairs becomes so large that pairing correlations are expected to play a minor role. At this regime the collective motion of a system should be strongly influenced by diabatic effects (level crossings) giving rise to large deformation changes [106].

\section{Shell Structure}

It was early realized that the symmetry-breaking mechanism is ultimately related to the behavior of the single-particle level density $g(e)$ of the intrinsic Hamiltonian:

$$
g(e)=\frac{d N}{d e} .
$$


( $g(e)$ is simply the number of states per unit of energy.) The atomic nucleus is expected to be more bound if the level density near the Fermi level is low. In particular, the nuclear ground state should correspond to the lowest possible degeneracy. Exceptionally stable systems (magic nuclei) are those with the least degenerate single-particle level density around the Fermi level, see Fig. 16.

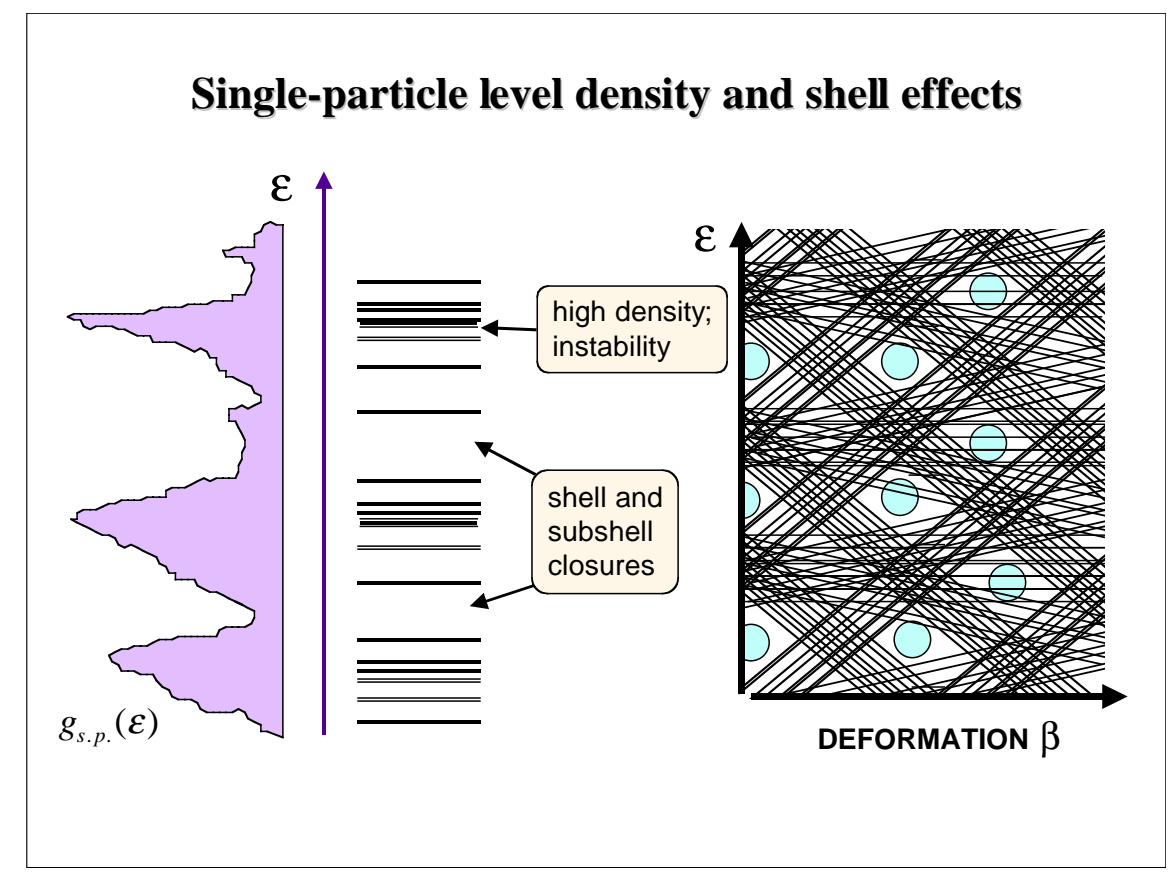

Fig. 16. Relation between single-particle level density and shell effects. Left: singleparticle levels and the corresponding level density. The regions of shell and subshell closures correspond to reduced $g(e)$. Right: schematic Nilsson diagram illustrating the appearance of spherical and deformed gaps in the single-particle spectrum.

The nuclear shell model describes well all nuclear properties that depend on the individual nucleonic motion in the vicinity of the Fermi surface. It is not possible, however, to apply the shell model alone to calculate bulk nuclear properties like binding energies, fission barriers, etc. It is because the single-particle shellmodel energy differs from the full HF energy by the residual-interaction term. On the other hand, the shell-model energy contains the two main elements of the symmetry-breaking mechanism, i.e., the single-particle level density and the perturbation due to the particle-vibration interaction. The deformation-driving force of the single-particle model can thus be estimated by means of the shellcorrection method developed by Swiatecki [115] and Strutinsky and collaborators [116-119]. The main assumption of the shell-correction approach is that the total 
energy of a nucleus can be composed of two parts,

$$
E=E^{\text {macr }}+E^{\text {shell }}
$$

where $E^{\text {macr }}$ is the macroscopic energy (smoothly depending on the number of nucleons and thus associated with the "uniform" distribution of single-particle orbitals) and $E^{\text {shell }}$ is the microscopic shell-correction term fluctuating with particle number and reflecting the non-uniformities of the single-particle level distribution.

For nuclei in which the surface energy dominates, the macroscopic energy tends to favor spherical shapes. The deformation-driving force is, therefore, contained in the shell-correction term, which can be approximated by

$$
E^{s h e l l}=\sum_{i=1}^{A} e_{i}-\sum_{i=1}^{\infty} n_{i} e_{i}
$$

where the average single-particle occupation numbers $n_{i}$ vary smoothly from 0 to 1 in the energy interval of the order of the energy difference between major shells. The approximation of the total energy by means of Eqs. (19) and (20) has a simple and elegant justification by means of the energy theorem formulated by Strutinsky $[116,117,119]$.

The behavior of $E^{\text {shell }}$ can be easily understood for any many-body system of fermions that generates average field. Indeed, if the level density around the Fermi level is large (level bunching, see Fig. 16), then the second term in Eq. (20) representing the smooth distribution of levels dominates (on the average it contains more occupied bound states) and $E^{\text {shell }}$ is large and positive. The opposite is expected in the case of the low single-particle level density: for magic nuclei $E^{\text {shell }}$ is large in magnitude and negative, thus leading to a strong stabilization of the total energy.

In order to understand the global features of shell-induced shape-polarization forces, it is instructive to plot the shell correction versus the number of particles and selected deformation parameters. A representative example is shown in Fig. 17 which displays the neutron shell-correction landscape of the modified harmonic oscillator potential (Nilsson potential) as a function of neutron number and quadrupole deformation $\varepsilon$. The topology of $E^{\text {shell }}$ changes periodically with particle number. The lowest shell energy is expected in the regions of low singleparticle level density, e.g., at spherical shapes for the magic numbers 20, 50, 82, and 126 . However, when going away from these magic numbers, the sphericalshape level density becomes large [ $(2 j+1)$-fold spherical degeneracy] and shell correction induces the strong quadrupole polarization towards deformed shapes.

The variation in the single-particle level density with shell filling, the level bunching, and the very existence of spherical and deformed magic numbers has a beautiful interpretation in terms of periodic orbits [75,121-123] in the corresponding classical problem. Indeed, the single-particle level density $g(\epsilon)=\sum_{i} \delta(\epsilon-$ $\left.\epsilon_{i}\right)$ can be represented by means of the Gutzwiller trace formula [124]

$$
g(\epsilon)=\tilde{g}(\epsilon)+\sum_{L} \sum_{k=1}^{\infty} a_{L k}(\epsilon) \cos \left[k\left(S_{L}(\epsilon) / \hbar-\frac{\pi}{2} \mu_{L}\right)\right],
$$




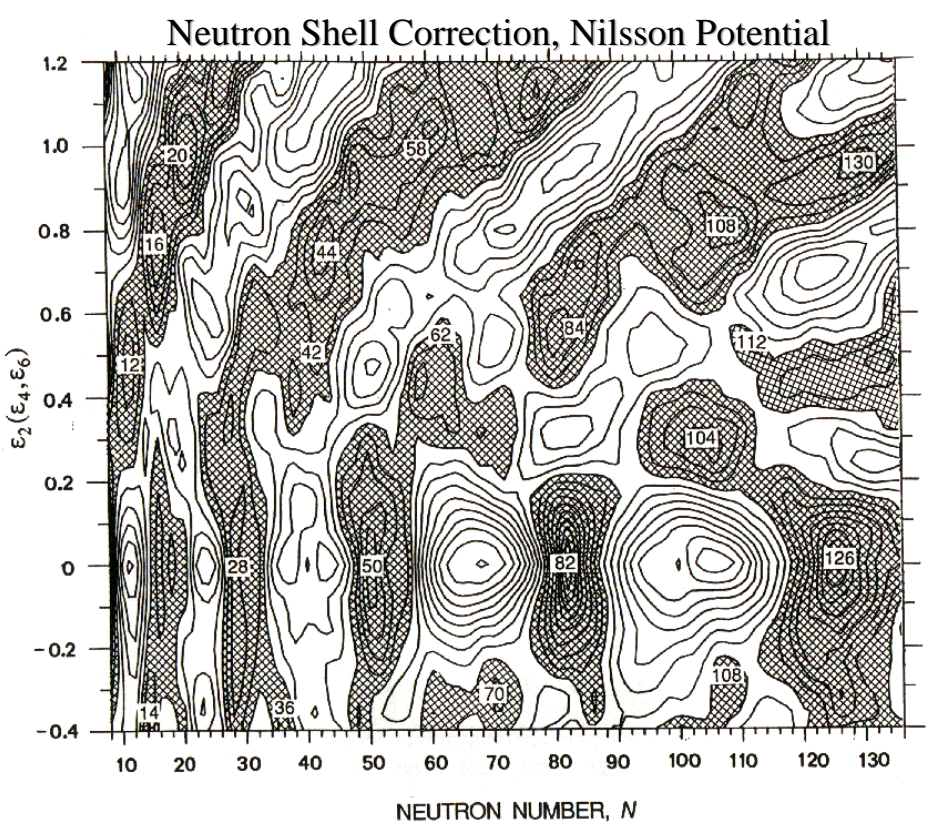

Fig. 17. Shell energy diagram in the $\left(\varepsilon_{2}, N\right)$ plane. The figure is taken from Ref. [120].

where $\tilde{g}$ is the average level density associated with $E^{\text {macr }}$, while the second term in Eq. (21) is the fluctuating part of $g$ responsible for shell effects. The shell energy can then be expressed as a sum over periodic orbits $L$. ( $S_{L}$ is the action integral associated with the orbit $L$ and $\mu_{L}$ is the Maslov index.) Consequently, the shell structure of the many-body system (hence the presence or absence of deformation) has its deep roots in the non-linear dynamics of the corresponding classical Hamiltonian and the geometry of classical orbits [123]. The microscopic analysis of the link between the phenomenon of spontaneous symmetry-breaking and the non-linear dynamics (and chaos) is one of the most vigorously pursued recent avenues in theoretical nuclear physics. Among many examples of such analyses are: explanation of supershell effects in superdeformed nuclei $[75,123]$ and discussion of shell structure in nuclei with permanent octupole deformations [125].

In the deformed shell model the important building block is the short-range pairing interaction. This is often approximated by means of the state-independent seniority force with the strength $G$. The macroscopic part of the total energy already contains the average pairing energy, which accounts for the main part of the even-odd mass difference. Therefore, it is the fluctuating part of the pairing energy, $\delta E^{\text {pair }}$, that gives an additional contribution to the total shell energy. The pairing field depends on the level density around the Fermi level, $g\left(e_{F}\right)$, 
as $[126]$

$$
\Delta \propto \exp \left[-\frac{1}{G g\left(e_{F}\right)}\right] .
$$

In the case of a large single-particle level density, $E^{\text {shell }}$ is positive but $\delta E^{\text {pair }}$ is large and negative (large $\Delta$ ). The opposite is true for systems with small $g\left(e_{F}\right)$, i.e., around large shell gaps. The cancellation between the shell energy and the pairing energy is yet another manifestation of the fact that pairing is the symmetry-restoring interaction. Indeed, equilibrium deformations calculated with the presence of pairing correlations are always reduced as compared to the situation without pairing.

\subsection{Examples of Nuclear Deformations}

It has been early recognized that various nuclear collective modes and deformations could be attributed to specific symmetries of the single-particle Hamiltonian and residual interaction. Indeed, as noted in Ref. [127]: "The recognition of the deformation and its degree of symmetry-breaking as the central element in defining rotational degrees of freedom opens new perspectives for generalized rotational spectra associated with deformations in many different dimensions including spin, isospin, and gauge spaces, in addition to the geometrical space of our classical world. The resulting rotational band structure may involve comprehensive families of states labeled by the different quantum numbers of the internally broken symmetries."

There are many examples of nuclear deformations [128]. The most common are the isoscalar shape deformations. Most nuclei have shapes well described by the even-parity multipole moments (quadrupole, hexadecapole,...). Extreme quadrupole deformations have been observed in superdeformed configurations such as those shown in Figs. 10 and 12. The dramatic elongations of superdeformed states, having a very different intrinsic structure than ground-states, are excellent examples of the Jahn-Teller effect in excited (many-particle, many-hole) configurations.

It has become clear during the last few years that certain nuclei can be described in terms of intrinsic shapes with parity-breaking (odd- $\lambda$ ) static moments. Stable reflection-asymmetric deformation in the body-fixed frame can be attributed to octupole interaction which couples intrinsic states of opposite parity. The regions of nuclei with strong octupole correlations correspond to particle numbers around $34,56,88$, and 134 , i.e. where the maximum $\Delta N=3$ octupole coupling occurs between the unique-parity subshell $(\ell, j)$ and the normalparity subshell $(\ell-3, j-3)$. Experimentally, for the Ra-Th $(\mathrm{Z} \sim 88, \mathrm{~N} \sim 134)$ and Ba-Sm (Z 56, N 88) nuclei, low-lying negative-parity states, parity doublets, and alternating parity bands with enhanced E1 transitions have been established (see Ref.[129]). Figure 18 shows examples of rotational bands corresponding to quadrupole and octupole-deformed nuclear rotors.

Deformations can show up in the context of interactions other than the multipole forces. For example, for strong pairing forces, there appear deformations 


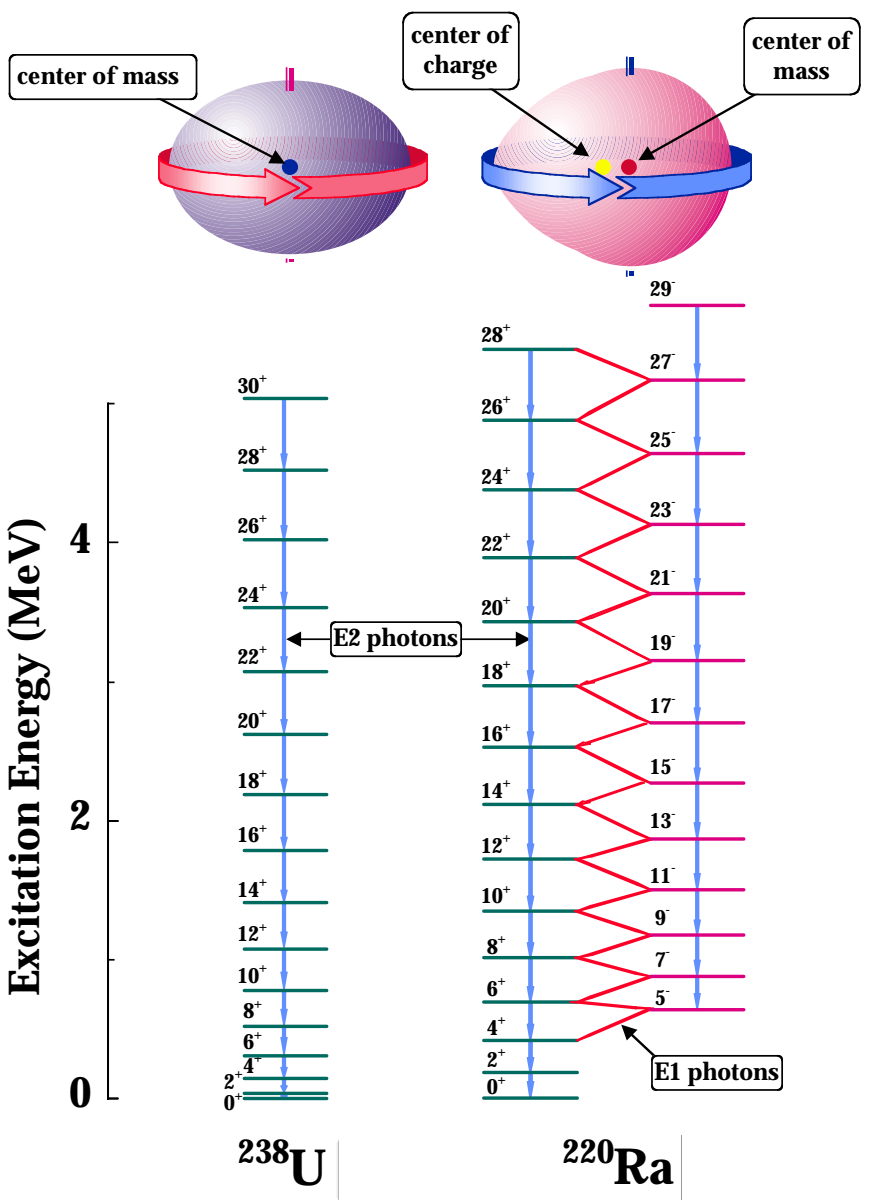

Fig. 18. Examples of nuclear collective bands. Left: ground-state band of quadrupoledeformed nucleus ${ }^{238} \mathrm{U}$. Right: alternating-parity ground-state band of octupoledeformed nucleus ${ }^{220} \mathrm{Ra}$.

that can be associated with static pairing gaps defining the orientation of a nucleus in the gauge space. Here, the broken symmetry is particle number; the intrinsic wave function is not an eigenstate of the particle number operator. Interesting pairing deformations are expected in N Z nuclei. Strong n-p correlations are likely to produce a static moment that will lead to spontaneous breaking of isospin. The elementary excitations of such systems are represented by quasi-particles which are mixtures of protons and neutrons [130].

Interesting new deformations can appear at high-spin states. The cranking Hamiltonian, $\boldsymbol{-} \boldsymbol{\omega} \boldsymbol{j}$, breaks the time-reversal symmetry in the rotating reference 
frame. In this context, one can talk about tilted rotation (rotation around an axis which is not a principal axis of a nucleus; the reorientation angles can be considered as collective parameters) or spontaneous breaking of signature quantum number [131-134].

\section{Far From Stability}

The uncharted regions of the $(N, Z)$ plane contain information that can answer many questions of fundamental importance for nuclear physics: How many protons and neutrons can be clustered together by the strong interaction to form a bound nucleus? What are the proton and neutron magic numbers in the neutronrich environment? What is the effective nucleon-nucleon interaction in a weakly bound nucleus? What are the phases of nucleonic matter? There are also related questions in the field of nuclear astrophysics. Since radioactive nuclei are produced in many astrophysical sites, knowledge of their properties is crucial to the understanding of the underlying processes. Today, the physics associated with radioactive nuclear beams (RNB) is one of the major thrusts of nuclear science worldwide.

From a theoretical point of view, exotic nuclei far from stability offer a unique test of those components of effective interactions that depend on the isospin degrees of freedom. Since the effective interaction in heavy nuclei has been adjusted to stable nuclei and to selected properties of infinite nuclear matter, it is by no means obvious that the isotopic trends far from stability, predicted by commonly used effective interactions, are correct. In models aiming at such an extrapolation, the important questions asked are: What is the density dependence of the two-body central force? What is the $\mathrm{N} / \mathrm{Z}$ dependence of the one-body spin-orbit force? What is the form of pairing interaction in weakly bound nuclei? What is the role of the medium effects and of the core polarization in the nuclear exterior (halo or skin region) where the nucleonic density is small? Similar questions are asked in connection with properties of nuclear matter, neutron droplets, and the physics of the neutron-star crust.

In many respects, weakly bound nuclei are much more difficult to treat theoretically than well-bound systems [135]. Hence, before tackling the problem of force parametrization at the extremes, one should be sure that the applied theoretical tools of the nuclear many-body problem are appropriate. The main theoretical challenge is the correct treatment of the particle continuum. For weakly bound nuclei, the Fermi energy lies very close to zero, and the decay channels must be taken into account explicitly. As a result, many cherished approaches of nuclear theory such as the conventional shell model, the pairing theory, or the macroscopic-microscopic approach must be modified. But there is also a splendid opportunity: the explicit coupling between bound states and continuum, and the presence of low-lying scattering states invite strong interplay and cross-fertilization between nuclear structure and reaction theory. Many methods developed by reaction theory can now be applied to structure aspects of 
loosely bound systems. Here, the representative example is the recent continuum shell-model description of the ${ }^{16} \mathrm{O}(\mathrm{p}, \gamma){ }^{17} \mathrm{~F}$ capture reaction [136].

A significant new theme concerns shell structure near the particle drip lines. Since the isospin dependence of the effective $N N$ interaction is largely unknown, the structure of single-particle states, collective modes, and the behavior of global nuclear properties is very uncertain in nuclei with extreme $N / Z$ ratios. For instance, some calculations predict [135] that the shell structure of neutron drip-line nuclei is different from what is known around the beta-stability valley. According to other calculations [137], a reduction of the spin-orbit splitting in neutron-rich nuclei is expected.

Correlations due to pairing, core polarization, and clustering are crucial in weakly bound nuclei. In a drip-line system, the pairing interaction and the presence of skin excitations (soft modes) could invalidate the picture of a nucleon moving in a single-particle orbit $[138,139,54,140,141]$. It is expected that the low-l spectroscopic strength is dramatically broadened when approaching the neutron drip line $[142,143]$. In addition, since the energy of the pigmy resonance in neutron-rich nuclei is close to the neutron separation energy, the presence of soft vibrational modes is also important in the context of the astrophysical r-process [144].

A fascinating aspect of halos and skins is the presence of clustering at the nuclear ground state. It is worth noting that all known neutron halo nuclei can be described in terms of cluster structures consisting of alpha particles surrounded by neutrons. The nuclear matter calculations indicate (see, e.g., Refs. $[145,146]$ ) the presence of deuteron and alpha condensates at low densities. This suggests that the transition from a mean-field regime (corresponding to the two-fluid proton-neutron system) to the limit of weak binding (characteristic of drip-line nuclei) does not have to be smooth. Most likely, one will encounter an intermediate phase corresponding to the presence of granularities (i.e., cluster structures) in the skin region.

On the proton-rich side, recent highlights are the discovery [147] of the twoproton unbound doubly magic nucleus ${ }^{48} \mathrm{Ni}$, the first (indirect) data on the core-breaking excitations in ${ }^{100} \mathrm{Sn}$ through the high-spin studies of ${ }^{99} \mathrm{Cd}$ (Ref. [148]), and studies of deformed proton emitters [149,150]. For a comprehensive review of challenges and opportunities in nuclear structure far from stability, I would like to refer the reader to the recent RIA White Paper [66] where many delightful examples can be found.

\section{Nucleus as a Finite Many-Body System}

The atomic nucleus is a complex, finite many-fermion system of particles interacting via a complicated effective force which is strongly influenced by in-medium effects. As such, it shows many similarities to other many-body systems involving many degrees of freedom, such as molecules, clusters, grains, mesoscopic rings, quantum dots, atom condensates, and others. There are many topics that are common to all these aggregations: existence of shell structure and collective 
modes (e.g., vibrations in nuclei, molecules, and clusters; superconductivity in nuclei and grains), various manifestations of the large-amplitude collective motion (such as multidimensional tunneling, coexistence, and phase transitions), nonlinear phenomena (many-fermion systems are wonderful laboratories to study chaos), and the presence of dynamical symmetries.

Historically, many concepts and tools of nuclear structure theory were brought to nuclear physics from other fields. Today, thanks to the wide arsenal of methods, many ideas from nuclear physics have been applied to studies of other complex systems. There are many splendid examples of such interdisciplinary research: studies of the multidimensional tunneling and of the large-amplitude collective motion and symmetry-breaking in many-body systems, applications of the nuclear mean-field theory and its extensions to studies of static and dynamical properties of metal clusters [151,152], use of symmetry-dictated approaches to describe collective excitations of complex molecules [153], studies of supersymmetries in many-body systems [154-156], applications of the nuclear random matrix theory to various phenomena in mesoscopic systems [157-159], studies of Bose condensates [160-162], and the description of correlations in many-fermion systems [163].

A beautiful example of physics on the borderline is the treatment of finitesize effects in the description of superconductivity of ultrasmall grains [164]. As in nuclei, superconducting grains exhibit the presence of the energy gap in the spectrum, and they show the odd-even staggering of binding energies due to a blocking effect caused by the presence of an odd electron [165-168]. As seen in Fig. 19, in the presence of an external magnetic field, quasi-particle spectra of grains strongly resemble those of rotating nuclei.

A topic of great interest is the signature of classical chaos in the associated quantum system, a sub-field known as quantum chaos. A nuclear physics theory (random matrix theory), developed in the 1950s and 60s to explain the statistical properties of the compound nucleus in the regime of neutron resonances [169], is now used to describe the universality of quantum chaos. Today, the random matrix theory is the basic tool of the interdisciplinary field of quantum chaos, and the atomic nucleus is still a wonderful laboratory of chaotic phenomena. Other excellent examples of interplay between chaotic and ordered motion in nuclei are parity-violation effects amplified by the chaotic environment [170], the appearance of very excited nuclear states (symmetry scars) well characterized by quantum numbers [171], and the appearance of collectivity in the many-body system governed by random two-body interactions [172-174].

The study of collective behavior, of its regular and chaotic aspects, is the domain where the unity and universality of all finite many-body systems is beautifully manifested. 


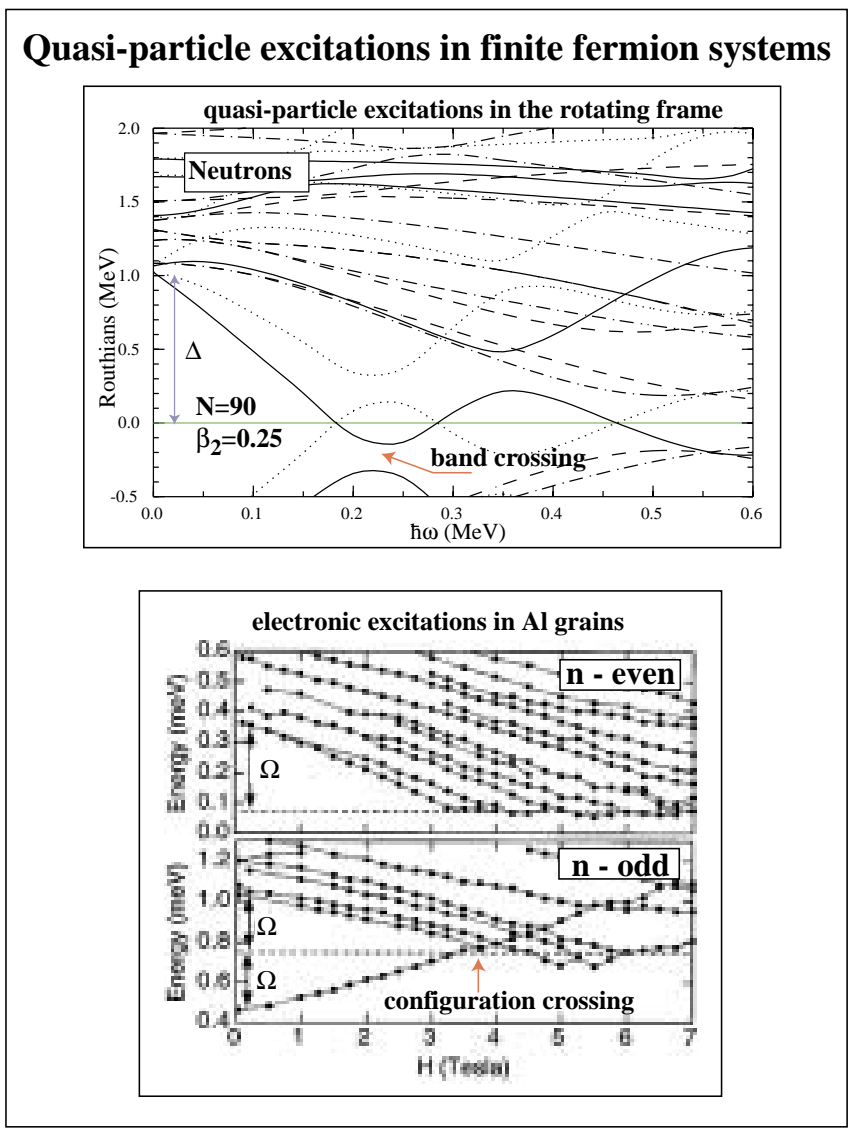

Fig. 19. Top: Quasi-particle neutron spectrum of rotating ${ }^{160} \mathrm{Yb}$ as a function of rotational frequency $\omega$ calculated within the Woods-Saxon cranking model assuming constant pairing gap $\Delta=1 \mathrm{MeV}$ and quadrupole deformation $\beta_{2}=0.25$. Bottom: Measured electronic excitations in Al grains as functions of the external magnetic field $H$ (from Ref. [164]).

\section{Summary}

In years to come, we shall see substantial progress in our understanding of nuclear structure - a rich and many-faceted field. An important element in this task will be to extend the study of nuclei into new domains.

There are many frontiers of today's nuclear structure. For very light nuclei, one such frontier is physics at subfemtometer distances where the internal quarkgluon structures of nucleons overlap. For heavier nuclei, the frontiers are defined 
by the extremes of the $N / Z$ ratio, atomic charge and nuclear mass, and angular momentum. The journey to "the limits" is a quest for new and unexpected phenomena which await us in the uncharted territory. However, the new data are also expected to bring qualitatively new information about the effective $N N$ interaction and hence about the fundamental properties of the nucleonic manybody system. By exploring exotic nuclei, one can magnify certain terms of the Hamiltonian which are small in "normal" nuclei, thus difficult to test. The hope is that after probing nuclear properties at the extremes, we can later improve the description of normal nuclei (at ground states, close to the valley of beta stability, etc.).

There are many experimental and theoretical suggestions pointing to the fact that the structure of exotic nuclei is different from what has been found in normal systems. New RNB facilities, together with advanced multi-detector arrays and mass/charge separators, will be essential in probing nuclei in new domains. The field is extremely rich and has a truly multidisciplinary character. Experiments with radioactive beams will make it possible to look closely into many exciting aspects of the nuclear many-body problem. A broad international community is enthusiastically using existing RNB facilities and hoping and planning for future-generation tools.

Advances in computer technology and theoretical modeling will make it possible to (i) better understand the bare $N N$ interaction in terms of quarks and gluons, and effective interactions in complex nuclei in terms of bare forces, and to (ii) answer fundamental questions concerning nuclear dynamics. These questions on the microscopic mechanism behind the small- and large-amplitude collective motion, on the impact of the Pauli principle on nuclear collectivity, and on the origin of short-range correlations have interdisciplinary character. Particularly strong are overlaps between nuclear structure and condensed matter physics (many-body methods, superconductivity, cluster physics, physics of mesoscopic systems), atomic and molecular physics (treatment of correlations, physics of particle continuum, dynamical symmetries), nonlinear dynamics (chaotic phenomena, large-amplitude collective motion), astrophysics (nucleosynthesis, neutron stars, supernovae), fundamental symmetries physics, and, of course, computational physics. The nuclear many-body system has it all!

\section{Acknowledgments}

This research was supported by the U.S. Department of Energy under Contract Nos. DE-FG02-96ER40963 (University of Tennessee) and DE-AC05-00OR22725 with UT-Battelle, LLC (Oak Ridge National Laboratory).

\section{References}

1. W. Nazarewicz, Nucl. Phys. A630, 239c (1998).

2. Nuclear Physics, the Core of Matter, the Fuel of Stars (National Academy Press, Washington, D.C. 1999); http://www.nas.edu/bpa/reports/reports.html. 
3. P.J. Woods and C.N. Davids, Ann. Rev. Nucl. Part. Sci. 47, 541 (1997).

4. B.S. Pudliner, A. Smerzi, J. Carlson, V.R. Pandharipande, S.C. Pieper, and D.G. Ravenhall, Phys. Rev. Lett. 76, 2416 (1996).

5. A. Smerzi, D.G. Ravenhall, and V.R. Pandharipande, Phys. Rev. C56, 2549 (1997).

6. M.V. Stoitsov, J. Dobaczewski, P. Ring, and S. Pittel, Phys. Rev. C 61, 034311 (2000).

7. C.J. Pethick and D.G. Ravenhall, Annu. Rev. Nucl. Part. Sci. 45, 429 (1995).

8. R. Machleidt, F. Sammarruca, and Y. Song, Phys. Rev. C53, 1483 (1996).

9. R. Machleidt, Proc. Int. Conf. Nuclear Structure'98, Gatlinburg 1998; nuclth/9809069.

10. V.G.J. Stoks, R.A.M. Klomp, C.P.F. Terheggen, and J.J. de Swart, Phys. Rev. C49, 2950 (1994).

11. R. Vinh Mau, this volume.

12. R.B. Wiringa, V.G.J. Stoks, and R. Schiavilla, Phys. Rev. C51, 38 (1995).

13. K. Maltman and N. Isgur, Phys. Rev. D29, 952 (1984).

14. T. Barnes, S. Capstick, M.D. Kovarik, and E.S. Svanson, Phys. Rev. C48, 539 (1993).

15. R.B. Wiringa, S.C. Pieper, J. Carlson, and V.R. Pandharipande, Phys. Rev. C 62, 014001 (2000).

16. S.C. Pieper, private communication 2000.

17. S.C. Pieper, R.B. Wiringa, and V.R. Pandharipande, Phys. Rev. C46, 1741 (1992).

18. J.G. Zabolitzky, Nucl. Phys. A228, 272 (1974).

19. J.G. Zabolitzky, Phys. Lett. 100B, 5 (1981).

20. K. Emrich and J.G. Zabolitzky, Nucl. Phys. A351, 439 (1981).

21. R.F. Bishop, E. Buendia, M.F. Flynn, and R.Guardiola, J. Phys. G17, 857 (1991).

22. J. Heisenberg and B. Mihaila, Phys. Rev. C59, 1440 (1999).

23. J.H. Heisenberg and B. Mihaila, Phys. Rev. Lett. 84, 1403 (2000).

24. R. Bieber, W. Glockle, J. Golak, M.N. Harakeh, D. Huber, H. Huisman, N. Kalantar-Nayestanaki, H. Kamada, J.G. Messchendorp, A. Nogga, H. Sakai, N. Sakamoto, M. Seip, M. Volkerts, S.Y. van der Werf, and H. Witala, Phys. Rev. Lett. 84, 606 (2000).

25. H. Witała, H. Kamada, A. Nogga, W. Glockle, Ch. Elster, and D. Huber, Phys. Rev. C59, 3035 (1999).

26. K.A. Brückner, in The Many Body Problem ed. by C. DeWitt (Wiley, New York, 1959), p. 47.

27. A.L. Fetter and J.D. Walecka, Quantum Theory of Many-Particle Systems (McGraw-Hill, Boston, 1971).

28. P. Ring and P. Schuck, The Nuclear Many-Body Problem (Springer-Verlag, Berlin, 1980).

29. J.P. Elliott, Proc. Roy. Soc. (London) A245, 128, 562 (1958).

30. A. Arima, F. Iachello, Phys. Lett. 57B, 39 (1975); Phys. Rev. Lett. 35, 1069 (1975).

31. F. Iachello and I. Talmi, Rev. Mod. Phys. 59, 339 (1987).

32. R.F. Casten and D.D. Warner, Rev. Mod. Phys. 60, 389 (1988).

33. Contemporary Concepts in Physics, vol. 6, Algebraic Approaches to Nuclear Structure, R.F. Casten, P.O. Lipas, D.D. Warner, T. Otsuka, K. Heyde, and J.P. Draayer (Harwood Academic Publ., New York, 1993).

34. P. Navratil, J.P. Vary, and B.R. Barrett, Phys. Rev. Lett. 84, 5728 (2000). 
35. W.C. Haxton and C.-L. Song, Phys. Rev. Lett. 84, 5484 (2000).

36. W.C. Haxton and T. Luu, nucl-th/0101022.

37. M.J. Savage and B.R. Holstein, nucl-th/0012060.

38. K. Langanke and A. Poves, Nucl. Phys. News 10, 3 (2000).

39. R.R. Whitehead and A. Watt, Phys. Lett. 35B, 189 (1971).

40. G. Martinez-Pinedo, A.P. Zuker, A. Poves, and E. Caurier, Phys. Rev. C55, 187 (1997).

41. A. Novoselsky and M. Vallieres, Phys. Rev. C57, R19 (1998).

42. P. Navratil and B.R. Barrett, Phys. Rev. C57, 562 (1998); Phys. Rev. C57, 3119 (1998) .

43. A. Holt, T. Engeland, M. Hjorth-Jensen, and E. Osnes, Phys. Rev. C 61, 064318 (2000).

44. E. Caurier, K. Langanke, G. Martinez-Pinedo, and F. Nowacki Nucl. Phys. A 653, 439 (1999).

45. G. Martinez-Pinedo, K. Langanke, and D.J. Dean, Astrophys. J. Suppl. Ser. 126, $493(2000)$.

46. A. Poves, J. Sanchez-Solano, E. Caurier, and F. Nowacki, nucl-th/0012077.

47. G. Martinez-Pinedo, P. Schwerdtfeger, E. Caurier, K. Langanke, W. Nazarewicz, and T. Sohnel, nucl-th/0101026.

48. A. Petrovici K.W. Schmidt, and A. Faessler, Z. Phys. A359, 19 (1997).

49. E. Hammaren, K.W. Schmid, and A. Faessler, Eur. Phys. J. A 2, 371 (1998).

50. S.E. Koonin, D.J. Dean, and K. Langanke, Phys. Rep. 278, 1 (1997); Ann. Rev. Nucl. Part. Sci. 47, 463 (1997).

51. D.J. Dean, S.E. Koonin, G.H. Lang, W.E. Ormand, and P.B. Radha, Phys. Lett. 317B, 275 (1993).

52. Y. Alhassid, G.F. Bertsch, D.J. Dean, and S.E. Koonin, Phys. Rev. Lett. 77, 1444 (1996).

53. M. Honma, T. Mizusaki, and T. Otsuka, Phys. Rev. Lett. 77, 3315 (1996).

54. T. Mizusaki, T. Otsuka, Y. Utsuno, M. Honma, and T. Sebe, Phys. Rev. C59, R1846 (1999).

55. S. Mizutori, J. Dobaczewski, G.A. Lalazissis, W. Nazarewicz, and P.-G. Reinhard, Phys. Rev. C 61, 044326 (2000).

56. C.J.G. Onderwater et al., Phys. Rev. Lett. 81, 2213 (1998).

57. R. Starink et al., Phys. Lett. 474B, 33 (2000).

58. C. Giusti, F.D. Pacati, K. Allaart, W.J.W. Geurts, W.H. Dickhoff, and H. Müther, Phys. Rev. C57, 1691 (1998).

59. J. Gomez del Campo, A. Galindo-Uribarri, J.R. Beene, C.J. Gross, J. F. Liang, M.L. Halbert, D.W. Stracener, D. Shapira, R.L. Varner, E. Chavez-Lomeli, and M. E. Ortiz, Phys. Rev. Lett. 86, 43 (2001).

60. L.V. Grigorenko, R.C. Johnson, I.G. Mukha, I.J. Thompson, and M.V. Zhukov, Phys. Rev. Lett. 85, 22 (2000).

61. J. Dobaczewski, W. Nazarewicz, and T.R. Werner, Z. Phys. A354, 27 (1996).

62. N. Tajima, S. Takahara, and N. Onishi, Nucl. Phys. A603, 23 (1996).

63. D. Hirata, K. Sumiyoshi, I. Tanihata, Y. Sugahara, T. Tachibana, and H. Toki, Nucl. Phys. A616, 438c (1997).

64. S.A. Fayans, S.V. Tolokonnikov, E.L. Trykov, and D. Zawischa, Nucl. Phys. A 676, 49 (2000).

65. F. Tondeur, S. Goriely, J.M. Pearson, and M. Onsi, Phys. Rev. C 62, 024308 (2000).

66. http://www.nscl.msu.edu/conferences/riaws00/ria-whitepaper-2000.pdf. 
67. Scientific Opportunities with Fast Fragmentation Beams from RIA, NSCL Report, March 2000. http://www.nscl.msu.edu/research/ria/whitepaper.pdf.

68. A. Arima, M. Harvey, and K. Shimizu, Phys. Lett. 30B, 517 (1969)

69. K.T. Hecht and A. Adler, Nucl. Phys. A137, 129 (1969).

70. J.N. Ginocchio, Phys. Rev. Lett. 78, 436 (1997).

71. J.N. Ginocchio and D.G. Madland, Phys. Rev. C57, 1167 (1998).

72. J. Meng, K. Sugawara-Tanabe, S. Yamaji, P. Ring, and A. Arima, Phys. Rev. C58, R628 (1998).

73. M. Göppert-Mayer, Phys. Rev. 75, 1969 (1949).

74. O. Haxel, J.H.D. Jensen, and H.E. Süss, Phys. Rev. 75, 1766 (1949).

75. A. Bohr and B.R. Mottelson, Nuclear Structure (Benjamin, New York, 1975), Vol. II.

76. P.K. Carroll and Kh. I. Hagim, Phys. Scr. 37, 682 (1988).

77. D. Pansegrau, P. Reiter, D. Schwalm, H. Bauer, J. Eberth, D. Gassmann, D. Habs, T. Hartlein, F. Kock, and H.G. Thomas, Phys. Lett. 484B, 1 (2000).

78. M.W. Drigert et al., Nucl. Phys. A515, 466 (1990).

79. M. Piiparinen, P. Kleinheinz, S. Lunardi, M. Ogawa, G. de Angelis, F. Soramel, W. Meczyński, and J.Blomqvist, Z. Phys. A337, 387 (1990).

80. G. Racah and I. Talmi, Physica 18, 1097 (1952).

81. M.A. Bentley et al. J. Phys. G17, 481 (1991).

82. P.J. Dagnall et al., Phys. Lett. 335B, 313 (1994).

83. J.L. Wood, K. Heyde, W. Nazarewicz, M. Huyse, and P. van Duppen, Phys. Rep. 215, 101 (1992).

84. K. Boretzky et al., Phys. Lett. 384B, 30 (1996).

85. W. Pauli, Naturwiss. 12 (1924) 741.

86. H. Schüler and Th. Schmidt, Z. Phys. 94, 457 (1935).

87. N. Bohr, Nature 137 (1936) 344.

88. N. Bohr and F. Kalckar, Mat. Fys. Medd. Dan. Vid. Selsk. 14, No. 10 (1937).

89. E. Teller and J.A. Wheeler, Phys. Rev. 53, 778 (1938).

90. L. Meitner and O.R. Frisch, Nature 143, 239 (1939).

91. N. Bohr and J.A. Wheeler, Phys. Rev. 56, 426 (1939).

92. J. Rainwater, Phys. Rev. 79, 432 (1950).

93. A. Bohr, Phys. Rev. 81, 134 (1951).

94. L.L. Foldy and F.J. Milford, Phys. Rev. 80, 751 (1950).

95. S. Gallone and C. Salvetti, Phys. Rev. 84, 1064 (1951).

96. S.G. Nilsson, Mat. Fys. Medd. Dan. Vid. Selsk. 29, No. 16 (1955).

97. S.A. Moszkowski, Phys. Rev. 99, 803 (1955).

98. K. Gottfried, Phys. Rev. 103, 1017 (1956).

99. A. Bohr, Mat. Fys. Medd. Dan. Vid. Selsk. 26, No. 14 (1952).

100. A. Bohr and B.R. Mottelson, Kg. Danske Videnskab. Selskab, Mat.-Fys. Medd. 27, No. 16 (1953).

101. D.L. Hill and J.A. Wheeler, Phys. Rev. 89, 1102 (1953).

102. H.A. Jahn and E. Teller, Proc. R. Soc. London, Ser. A 161, 220 (1937).

103. W. Nazarewicz, Int. J. Mod. Phys. E2, 51 (Supp. 1993).

104. W. Nazarewicz, Nucl. Phys. A574, 27c (1994).

105. M. Born and R.J. Oppenheimer, Ann. Physik (Leipzig) 89, 457 (1927).

106. W. Nazarewicz, Nucl. Phys. A557, 489c (1993).

107. P. Ring, Proc. Workshop on Microscopic Models in Nuclear Structure Physics, Oak Ridge 1988, ed. by M.W. Guidry et al., (World Scientific, 1988) p. 298.

108. A. Bulgac, Phys. Rev. C41, 2333 (1990). 
109. A. Klein and N.R. Walet, Phys. Rev. C48, 178 (1993).

110. A. Bulgac, Phys. Rev. Lett. 67, 965 (1991).

111. V.M. Strutinsky, Z. Phys. A280, 99 (1977).

112. P.-G. Reinhard and E.W. Otten, Nucl. Phys. A420, 173 (1984).

113. D. Schütte and J. Da Providencia, Nucl. Phys. A282, 518 (1977).

114. D.J. Thouless, Nucl. Phys. 22, 78 (1961).

115. W.J. Swiatecki, in: Proc. 2nd Int. Conf. on Nuclidic Masses, Vienna, 1963, ed. by: W.H. Johnson, Jr. (Springer-Verlag, Vienna 1964), p. 58.

116. V.M. Strutinsky, Nucl. Phys. A95, 420 (1967).

117. V.M. Strutinsky, Nucl. Phys. A122, 1 (1968).

118. G.G. Bunatian, V.M. Kolomietz and V.V. Strutinsky, Nucl. Phys. A188, 225 (1972).

119. M. Brack, J. Damgård, A.S. Jensen, H.C. Pauli, V.M. Strutinsky and C. Y. Wong, Rev. Mod. Phys. 44, 320 (1972).

120. I. Ragnarsson and R.K. Sheline, Phys. Scr. 29, 385 (1984).

121. R. Balian and C. Bloch, Ann. Phys. (NY) 69, 76 (1971).

122. V.M. Strutinsky and A.G. Magner, Sov. J. Part. Nucl. 7, 138 (1976).

123. V.M. Strutinsky, A.G. Magner, S.R. Ofengenden and T. Døssing, Z. Phys. A283, 269 (1977)

124. M.C. Gutzwiller, J. Math. Phys. 8 (1967) 1979; ibid 12, 343 (1971).

125. K. Arita and K. Matsuyanagi, Prog. Theor. Phys. 89, 389 (1993).

126. S.T. Belyaev, Mat. Fys. Medd. Dan. Vid. Selsk. 31 (No. 11) (1959).

127. A. Bohr, Rev. Mod. Phys. 48, 365 (1976).

128. W. Nazarewicz and I. Ragnarsson, in: CRC Handbook on Nuclear Decay Modes, 1993.

129. P. Butler and W. Nazarewicz, Rev. Mod. Phys. 68, 349 (1996).

130. S. Åberg, H. Flocard, and W. Nazarewicz, Ann. Rev. Nucl. Part. Sci. 40, 439 (1990).

131. S. Frauendorf, Nucl. Phys. A557, 259c (1993).

132. S. Frauendorf, Rev. Mod. Phys. (to be published).

133. S. Frauendorf, Nucl. Phys. A677, 115 (2000).

134. V.I. Dimitrov, S. Frauendorf, and F. Dönau, Phys. Rev. Lett. 84, 5732 (2000).

135. J. Dobaczewski and W. Nazarewicz, Phil. Trans. R. Soc. Lond. A 356, 2007 (1998).

136. K. Bennaceur, N. Michel, F. Nowacki, J. Okołowicz, and M.Płoszajczak, Phys. Lett. 488B, 75 (2000).

137. G.A. Lalazissis, D. Vretanar, W. Poschl, and P. Ring, Phys. Lett. 418B, 7 (1998).

138. J. Dobaczewski, W. Nazarewicz, T.R. Werner, J.-F. Berger, C.R. Chinn, and J. Dechargé, Phys. Rev. C53, 2809 (1996).

139. W. Pöschl, D. Vretenar, G.A. Lalazissis, and P. Ring, Phys. Rev. Lett. 79, 3841 (1997).

140. M. Bender, Phys. Rev. C 61, 031302 (2000).

141. F. Barranco, R.A. Broglia, G. Colo, and E. Vigezzi, nucl-th/0005073.

142. S.A. Fayans, S.V. Tolokonnikov, and D. Zawischa, Phys. Lett. 491B, 245 (2000).

143. H. Lenske, F. Hofmann, and C. Klein, these proceedings.

144. S. Goriely, Phys. Lett. 436B, 10 (1998).

145. G. Röpke, A. Schnell, P. Schuck, and P. Nozières, Phys. Rev. Lett. 80, 3177 (1998).

146. M. Beyer, S.A. Sofianos, C. Kuhrts, G. Röpke, and P. Schuck, Phys. Lett. 488B, 247 (2000). 
147. B. Blank et al., Phys. Rev. Lett. 84, 1116 (2000).

148. M. Lipoglavsek et al., to be published.

149. A.A. Sonzogni, C.N. Davids, P.J. Woods, D. Seweryniak, M.P. Carpenter, J.J. Ressler, J. Schwartz, J. Uusitalo, and W.B. Walters, Phys. Rev. Lett. 83, 1116 (1999).

150. A.T. Kruppa, B. Barmore, W. Nazarewicz, and T. Vertse, Phys. Rev. Lett. 84 4549 (2000)

151. W. Satuła, J. Dobaczewski, and W. Nazarewicz, Phys. Rev. Lett. 81, 3599 (1998).

152. G.F. Bertsch and K. Yabana, Nucl. Phys. A649, 423c (1999).

153. F. Iachello, Nucl. Phys. A560, 23 (1993).

154. A. Metz, J. Jolie, G. Graw, R. Hertenberger, J. Groger, C. Gunther, N. Warr, and Y. Eisermann, Phys. Rev. Lett. 83, 1542 (1999).

155. J. Groger, J. Jolie, R. Krucken, C.W. Beausang, M. Caprio, R.F. Casten, J. Cederkall, J.R. Cooper, F. Corminboeuf, L. Genilloud, G. Graw, C. Gunther, M. de Huu, A.I. Levon, A. Metz, J.R. Novak, N. Warr, and T. Wendel, Phys. Rev. C 62, 064304 (2000).

156. F. Iachello, these proceedings.

157. Y. Alhassid and H. Attias, Phys. Rev. Lett. 76, 1711 (1996).

158. Y. Alhassid, M. Gökcedag, and A.D. Stone, Phys. Rev. B 58, 7524 (1998).

159. T. Rupp, H.A. Weidenmüller, and J. Richert, Phys. Lett. 483B, 331 (2000); Erratum Phys. Lett. 484B, 376 (2000).

160. R. Balian, H. Flocard, and M. Vénéroni, cond-mat/9802006.

161. B. Mottelson, Phys. Rev. Lett. 83, 2695 (1999).

162. G.F. Bertsch and T. Papenbrock, Phys. Rev. Lett. 83, 5412 (1999).

163. J.N. Ginocchio and W.C. Haxton, Phys. Rev. Lett. 77, 1568 (1996).

164. D.C. Ralph, C.T. Black, and M. Tinkham, Phys. Rev. Lett. 78, 4087 (1997).

165. R. Rossignoli, N. Canosa, and P. Ring, Phys. Rev. Lett. 80, 1853 (1998).

166. J. Dukelsky and G.G. Dussel, Phys. Rev. C59, R3005 (1999).

167. R. Balian, H. Flocard, and M. Vénéroni, Phys. Rep. 317, 251 (1999).

168. G. Sierra, J. Dukelsky, G.G. Dussel, J. von Delft, and F. Braun, Phys. Rev. B 61, R11890 (2000).

169. F.J. Dyson and M.L. Mehta, J. Math. Phys. 4, 701 (1963).

170. H.A. Weidenmüller, Nucl. Phys. A574, 75c (1994).

171. H.A. Weidenmüller, Nucl. Phys. A520, 509c (1990).

172. C.W. Johnson, G.F. Bertsch, D.J. Dean, and I. Talmi, Phys. Rev. Lett. 80, 2749 (1998).

173. C.W. Johnson, G.F. Bertsch, D.J. Dean, and I. Talmi, Phys. Rev. C61, 014311 (2000).

174. D. Mulhall, A. Volya, V. Zelevinsky, Phys. Rev.Lett. 85, 4016 (2000). 